\title{
Tuning macroscopic sliding friction at soft contact interfaces: Interaction of bulk and surface heterogeneities
}

\author{
K.İ. Kılıç, İ. Temizer* \\ Department of Mechanical Engineering, Bilkent University, 06800 Ankara, Turkey
}

\section{A R T I C L E I N F O}

\section{Article history:}

Received 11 July 2016

Received in revised form

19 August 2016

Accepted 23 August 2016

Available online 25 August 2016

Keywords:

Soft interfaces

Finite deformations

Textured surfaces

Sliding friction

\begin{abstract}
A B S T R A C T
Macroscopic frictional response of soft interfaces is strongly governed by the interaction of surface heterogeneities such as micro-texture features with bulk heterogeneities such as voids or inclusions beneath the highly deformable surface. This microscopic interaction manifests itself on the macroscale as an interface response that is reminiscent of stick-slip. Consequently, the accompanying macroscopic friction signal exhibits strong oscillations around a mean value, which itself significantly differs from its microscopic value due to finite deformations. In this work, a mechanism is proposed which enables the tuning of the macroscopic friction signal of soft interfaces. Specifically, it is demonstrated that optimally positioning subsurface particles in the vicinity of micro-texture features can significantly reduce observed oscillations, thereby allowing control of macroscopic sliding friction.
\end{abstract}

(c) 2016 Elsevier Ltd. All rights reserved.

\section{Introduction}

\subsection{Motivation and goal}

Soft contact interfaces, where one or both of the interacting surfaces can deform significantly even at light loads, have an extremely rich frictional response. The underlying complexity in this response primarily arises from the interaction of random roughness or a periodic texture with surface adhesion and bulk viscoelasticity, possibly in the presence of an interfacial fluid. The physical characterization of soft interface friction is relevant to the technological applications of synthetic materials [1-3] as well as to have a better understanding of the natural functioning of biological tissue [4-6]. Overall, the typical characteristics of soft interface friction range from a considerable temperature-velocitypressure sensitivity $[1,7,8]$ to dynamic phenomena such as Schallamach waves $[9,10]$ and stick-slip behavior $[11,12]$.

The goal of this work is the numerical modeling and simulation of how macroscopic soft interface friction is influenced and can be controlled by the simultaneous presence and strong interaction of microscopic surface and bulk heterogeneities. In what follows, different aspects of this goal are individually reviewed. For this purpose, it is useful to consider the influence of heterogeneities from a broader interface mechanics perspective, by momentarily considering adhesion in addition to friction, however still maintaining a strict focus on soft interfaces. Also, in comparison to the

\footnotetext{
* Corresponding author.

E-mail address: temizer@bilkent.edu.tr (ì. Temizer).
}

choice of works cited above which are predominantly experimental, modeling studies with theoretical and computational approaches will be addressed as well.

\subsection{Microscopic surface and bulk heterogeneities}

Surface heterogeneities are typically characterized by an oscillatory boundary geometry or interfacial features whereas bulk heterogeneities are associated with oscillatory constitutive properties. The influence of surface heterogeneities on the interface response has been thoroughly investigated. Investigations towards the demonstration and modeling of this influence have culminated to a large body of work - see [13-16] in the context of adhesion and $[1,4,11,17,18]$ for friction regarding recent representative studies with both experimental and modeling aspects. The influence of bulk heterogeneities, mostly accompanied by the simultaneous presence of surface heterogeneities, is comparatively much less investigated. Now, it is well-known that the mixing of a base material with particulate or fibrous additives will display a modified interface response, exemplified by the engineering of the adhesive and viscous contributions to rubber friction [19-21]. Similarly, the frictional response of biological layered media such as skin $[4,5,22]$ and the adhesive response of synthetic media with a subsurface structure [23] are examples where the presence of bulk heterogeneity has a decisive influence on the interface response. In passing, similar influences for stiff materials and numerical studies which explicitly resolve the composite bulk structure to model this influence may be mentioned [24,25]. However, in all these examples, the interaction of the bulk heterogeneities with the interface is not strongly observable macroscopically. For instance, a 
mixture will effectively behave as a new homogeneous material which induces a quantitatively different but a qualitatively similar interface response.

As the particular case of interest in this study, a strongly observable qualitative difference in the macroscopic interface response due to the presence of bulk heterogeneities would be associated with the (dis)appearance of oscillations and peaks during peeling for adhesion or during sliding for friction. Indeed, such strong interactions between bulk heterogeneities and the interface have been observed and modeled for adhesion [26] and friction [10], in both cases with a periodic near-surface elastic property distribution. Oscillations are introduced into the macroscopic interface response in the former case, and existing ones due stickslip behavior are eliminated in the latter. In both cases, the influence of each bulk heterogeneity is clearly detectable and this influence directly correlates with its size. Present study concentrates on this class of bulk heterogeneities in the simultaneous presence of surface heterogeneities.

\subsection{Tuning macroscopic friction}

The aim in introducing bulk heterogeneities will be to influence the sliding friction response of the interface. From a macroscopic point of view, this response is reminiscent of stick-slip behavior. Stick-slip response at homogeneous soft interfaces, which may be desirable or undesirable, has been observed and analyzed for a long time. The signature of this response is a highly oscillatory friction signal $[9,11]$. It is now known that this behavior may be tuned by splitting the contact zone through the introduction of surface and bulk heterogeneities, modeling of which has also been recently attempted $[10,27,28]$. Specifically, tuning refers not only to an ability to turn the oscillations on or off [11] but also to an ability to gradually control the oscillation amplitude [10]. Now, when surface heterogeneities are introduced to both surfaces in contact, such as through texturing to engineer the macroscopic tribological response of the interface, these will also give rise to significant oscillations in the friction signal - a more specific example will be provided in the next section. These oscillations are not due to stick-slip behavior in the microscopic sense but rather due to the interaction between individual surface heterogeneities, which cause the interface to lock and then break free cyclically as the heterogeneities slide over each other. However, irrespective of the microscopic cause, the friction signal displays highly oscillatory characteristics. Since surface heterogeneities are the root cause of this behavior, the ability to control the macroscopic friction signal by tuning the amplitude of the oscillations can only be realized by introducing additional bulk heterogeneities that can strongly interact with the surface heterogeneities, which is to be pursued presently.

\subsection{Computational aspects of soft interface friction}

In common with most tuning mechanisms discussed earlier, a strong interaction between bulk and surface heterogeneities will rely on finite deformations that are typical of soft interfaces and therefore requires a numerical capability to address mechanics in this deformation regime. This numerical capability will rely on computational frameworks which have been developed in order to assess, qualitatively and quantitatively, the influence of surface heterogeneities such as roughness or texture on the dry sliding friction of soft contact interfaces. Although such frameworks have been developed and applied in a large number of studies, mostly to rubber friction, a significant portion of these are based on an infinitesimal deformation setting due to an ability to incorporate random roughness across multiple scales in a reliable manner see $[1,18,29,30]$ among others. There are also a limited number of works which aim to address the finite deformations sustained in the vicinity of the contact interface in a setting that can resolve the microscopic interactions and upscale them towards the macroscopic interface friction [31-38]. Finite deformations or, in general, the adoption of a materially and kinematically fully nonlinear setting for a faithful representation of soft interface mechanics may not always deliver significant improvements over an infinitesimal deformation setting, either in terms of microscopic measures such as the resolution of the contact interface or in terms of macroscopic ones such as a friction coefficient [39]. However, a number of studies strictly rely on finite deformations, such as for granular interfaces [35,36] and rough surfaces [32-34], since core observations stated therein regarding the influence of surface heterogeneities on the macroscopic response have been shown to vanish in the limit of infinitesimally small deformations. The present study is in the spirit of this latter class of studies where it is essential to have a nonlinear framework which can reflect the finite deformations within a boundary layer of the soft material in the vicinity of the contact interface in order to accurately capture bulk-surface heterogeneity interaction towards the tuning of the macroscopic friction signal.

\subsection{Outline}

The outline of this work is as follows. First, the computational framework with which all the numerical experiments are to be performed will be outlined in Section 2. Next, the individual influences of the particular bulk and surface heterogeneities considered in these experiments are characterized in Section 3. The central feature of bulk-surface heterogeneity interaction is identified in Section 4 and this feature is probed in Section 5 to demonstrate a mechanism for controlling the oscillations in the macroscopic friction signal while keeping its mean value virtually constant. A simple model for the functioning of this mechanism which can accelerate the tuning of the interface response by guiding it towards the desired signal is proposed in Section 6. The influences of major numerical parameters on the overall computational framework are outlined in Section 7, followed by an analysis of limiting cases with respect to material parameters and geometrical variables in Section 8. The study is finally concluded with a brief summary and an outlook.

\section{Computational setup and numerical parameters}

The numerical studies to be performed in this work are based on the computational homogenization framework for soft interface friction that was introduced in [33], where the details of the underlying numerical approach as well as a number of fundamental observations regarding multiscale contact at finite deformations may be found - see also [34] for an extension to a thermomechanical setting. In this section, major aspects of this framework which are important for the purposes of the present study are summarized and the fundamental micromechanical variables are introduced.

The default computational setup to be built upon consists of a microscale contact interface featuring two surfaces (Fig. 1(a)), which will be referred to as the slave $(s)$ and the master $(m)$ following standard computational contact mechanics terminology. The setup represents the microscopic contact conditions at a point across the macroscopic interface under large frictional slip. Consequently, the upper (master) surface slides over the comparatively much stiffer lower (slave) surface at a prescribed macroscopic contact pressure $(\bar{p})$ and slip velocity $(\bar{v})$. The direction of sliding is to the right in all computations. The parameters which control or influence the macroscopic contact response that is 


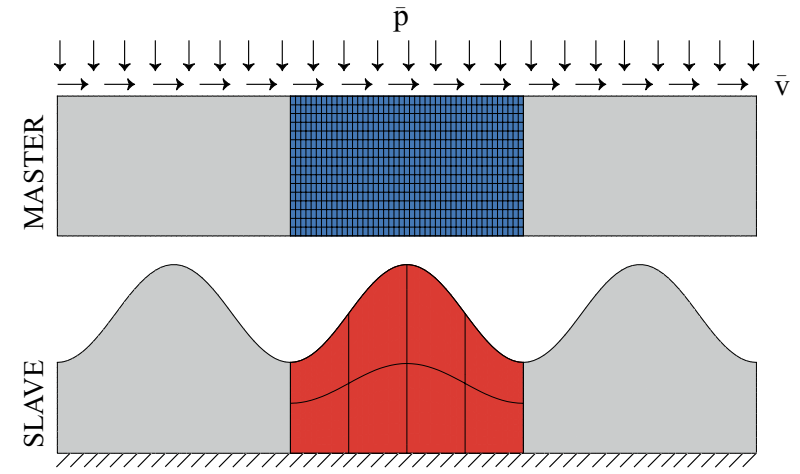

(a) Default computational setup

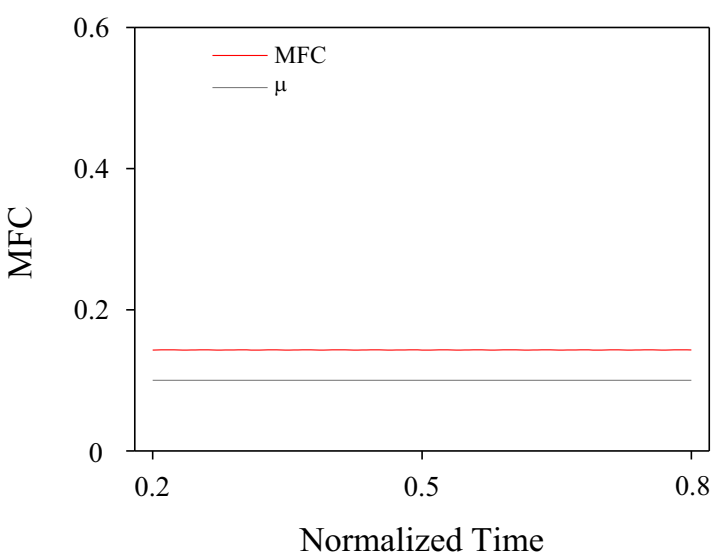

(b) MFC of the default setup from

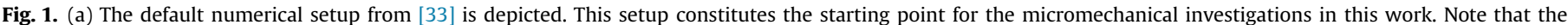

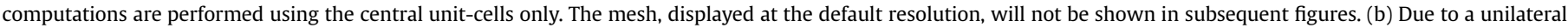
texture, a constant MFC signal (0.143) is observed which, however, is considerably larger than the microscopic friction coefficient ( $\mu=0.1$ ) due to finite deformations.

Table 1

Default values for the parameters of the micromechanical setup.

\begin{tabular}{|c|c|}
\hline Numerical parameters & Default values \\
\hline Microscopic friction coefficient $(\mu)$ & 0.1 \\
\hline Macroscopic contact pressure $(\overline{\mathrm{p}})$ & $1 \mathrm{MPa}$ \\
\hline Macroscopic sliding velocity $(\overline{\mathrm{v}})$ & $10 \mathrm{~m} / \mathrm{s}$ (fixed) \\
\hline Root-mean-square of the master $\left(\mathrm{RMS}_{\mathrm{m}}\right)$ & $0.04 \mu \mathrm{m}$ \\
\hline Root-mean-square of the slave $\left(\mathrm{RMS}_{\mathrm{s}}\right)$ & $0.3 \mu \mathrm{m}$ (fixed) \\
\hline Root-mean-square ratio $\left(\mathrm{RMS}_{\mathrm{r}}=\mathrm{RMS}_{\mathrm{m}} / \mathrm{RMS}_{\mathrm{s}}\right)$ & $1 / 7.5$ \\
\hline Shear moduli of slave $\left(G_{s}\right)$ & $5 \mathrm{MPa}$ \\
\hline Shear moduli of master $\left(G_{m}\right)$ & $0.625 \mathrm{MPa}$ \\
\hline Mismatch ratio for slave and master $\left(G_{s} / G_{m}\right)$ & 8 \\
\hline Shear moduli of particles $\left(G_{p}\right)$ & $5 \mathrm{MPa}$ \\
\hline Mismatch ratio for master and particles $\left(M R=G_{p} / G_{m}\right)$ & 8 \\
\hline Ratio $(\mathrm{K} / \mathrm{G})$ of bulk modulus $(\mathrm{K})$ to shear modulus $(\mathrm{G})$ & 12 (fixed) \\
\hline $\begin{array}{l}\text { Height }(\mathrm{H}) \text { (with respect to the mean of micro-texture, } \\
\text { if present) }\end{array}$ & $6 \mu \mathrm{m}$ \\
\hline Width $(\mathrm{W})$ & $10 \mu \mathrm{m}$ \\
\hline Oscillation Period $(T=W / \bar{v})$ & $1 \mu \mathrm{s}$ \\
\hline $\begin{array}{l}\text { Vertical Shift (d) (with respect to the default particle } \\
\text { position) }\end{array}$ & $0 \mu \mathrm{m}$ \\
\hline Volume Fraction of Particles (VF) & 0.25 \\
\hline Macroscopic friction coefficient (MFC) & $\begin{array}{l}0.143 \text { (non- } \\
\text { oscillatory) }\end{array}$ \\
\hline $\begin{array}{l}\text { Average friction coefficient (AFC) (Time average of } \\
\text { MFC) }\end{array}$ & 0.143 \\
\hline $\begin{array}{l}\text { Peak-to-peak macroscopic friction coefficient }\left(\mathrm{P}_{\text {to }} \mathrm{P}-\right. \\
\quad \mathrm{MFC}=\mathrm{Max} \text { MFC - Min MFC })\end{array}$ & 0 \\
\hline $\begin{array}{l}\text { Mesh resolution for the master (number of elements } \\
\text { along vertical: horizontal directions) }\end{array}$ & $45: 27$ \\
\hline $\begin{array}{l}\text { Mesh resolution for the slave (number of elements } \\
\text { along vertical: horizontal directions) }\end{array}$ & $4: 3$ \\
\hline
\end{tabular}

induced by this micromechanical setup are summarized in Table 1 along with their default values, which are chosen within physically meaningful ranges. The default values are chosen identical to those in [33] where possible in order to directly benefit from the conclusions stated therein and thereby avoid repeating various investigations which have already been extensively discussed.

In the default computational setup, the slave is assigned a periodic and sinusoidal micro-texture while the master is homogeneous both with respect to surface and the bulk, i.e. it has a flat surface and consists of a single material, thereby delivering a unilaterally micro-textured interface. Due to periodicity, a unit-cell of the two-body contact setup is easily identified, which consists of one texture period of the slave and a matching portion from the master. The height of the unit-cell for both surfaces is set to
$0.6 \mu \mathrm{m}$, which is measured with respect to the texture mean if the surface is non-flat, and the width is chosen as $1 \mu \mathrm{m}$. Purely elastic materials are considered in this work, by default of the Ogdentype. The shear modulus of the slave $\left(G_{s}\right)$ will be kept constant in the majority of the test cases. Therefore, the shear modulus of the master $\left(G_{m}\right)$ is indicated indirectly by the ratio $G_{s} / G_{m}$, the default value being 8 . The ratio of bulk to shear moduli of each surface will be kept at a constant value of 12 . The mesh resolution of the master is chosen much finer in comparison to the slave, which is stiffer and hence does not deform significantly. Higher-order B-splines are used for the discretization of both the bulk as well as the surface of the master and the slave, which decreases the number of elements needed for a given accuracy in comparison to standard finite elements. The mesh resolution is chosen to ensure converged numerical results. The underlying computational contact mechanics algorithm relies on a mortar-based discretization of the contact variables and the augmented Lagrangian technique for enforcing both normal and tangential contact constraints.

Modifications to this default unilaterally textured computational setup will mainly target the parameters which define the characteristics of the bulk and surface heterogeneities that will be additionally imposed on the master. Specifically, the surface heterogeneity will correspond to texture features such as asperities assigned to the master, thereby leading to a bilaterally textured interface, while the bulk heterogeneity will be associated with a circular particle embedded beneath the master surface, thereby leading to a composite master. In this context, the root-meansquare (RMS) of the texture will be controlled for the surfaces whereas the change in the particle property is reflected through variations in stiffness, volume fraction and position of the subsurface particle. More specifically, in the cases where a bilateral texture is assigned, the ratio $R M S_{\mathrm{r}}=\mathrm{RMS}_{\mathrm{m}} / \mathrm{RMS}_{\mathrm{s}}$ is changed while $\mathrm{RMS}_{\mathrm{S}}$ is kept constant. The default value of this parameter will be $\mathrm{RMS}_{\mathrm{r}}=1 / 7.5$, which is small yet sufficient to induce significant oscillations in the macroscopic friction response. The size of the particle within the master unit-cell is controlled by the volume fraction VF, which is set to 0.25 by default. It is assumed that the particle is perfectly bonded to the surrounding material. The stiffness mismatch ratio (MR) between the particle and the surrounding master matrix material is another major variable. $M R=1$ corresponds to the case where the particle is not distinguishable from the master, and larger/smaller values indicate stiffer/softer particles. In practice, the particle could be selected from a broad range of polymeric materials which are readily obtainable as 
spherical beads at various sizes. For the default choice, the particle is as stiff as the slave. Finally, the position of the particle is a major microscopic control parameter. In the horizontal direction, the position will be indicated through normalized coordinates where a value of -1 or 1 corresponds, respectively, to the case where the center of the particle overlaps with the left or right boundary of the master unit-cell.

The macroscopic contact response that is computed from this micromechanical setup is characterized through its macroscopic (sliding) friction signal. The contact interface of the two surfaces is assigned a constant microscopic friction coefficient, $\mu$, which is equal to 0.1 by default. The presence of bulk and surface heterogeneities modifies how this microscale frictional response is reflected onto the macroscale in the form a macroscopic friction

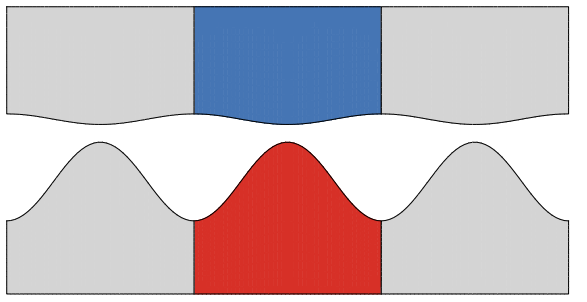

(a) Surface heterogeneity: Bilateral texture

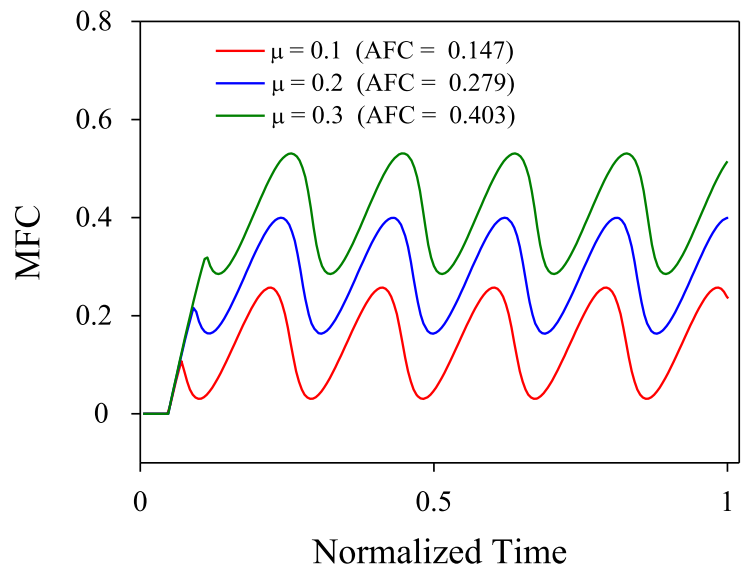

(c) MFC in setup (a) for varying $\mu$

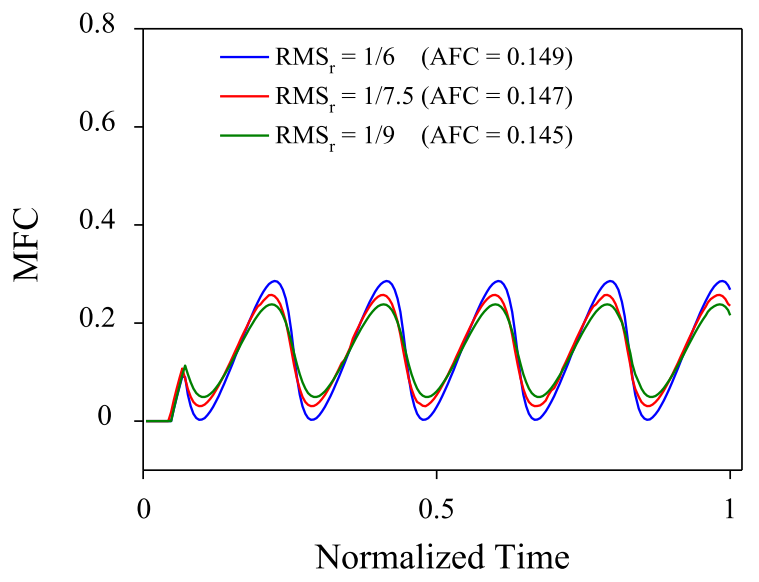

(e) MFC in setup (a) for varying $\mathrm{RMS}_{\mathrm{r}}$ coefficient (MFC), which is defined through the ratio of the tangential force to the normal force applied onto the micromechanical sample. Indeed, the default computational setup, which has a unilateral texture and a homogeneous master, generates a constant MFC $=0.143$ that is larger than $\mu=0.1$ - see Fig. 1 (b). Note that this is a strictly finite deformation effect that is characteristic of soft interface friction [32,33] where not only the out-of-plane but also the in-plane statistical characteristics of the texture can significantly change [40]. In other words, for this default setting, the macroscopic response would be identical to the microscopic one in the limit of infinitesimal deformations, for instance as the macroscopic contact pressure is significantly reduced. It is important to highlight that this statement also pertains to the majority of the investigations to be addressed in this work where the emphasis is

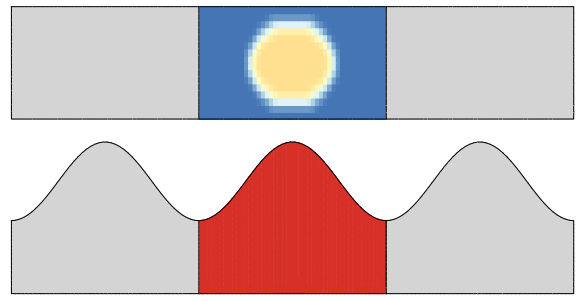

(b) Bulk heterogeneity: Composite master

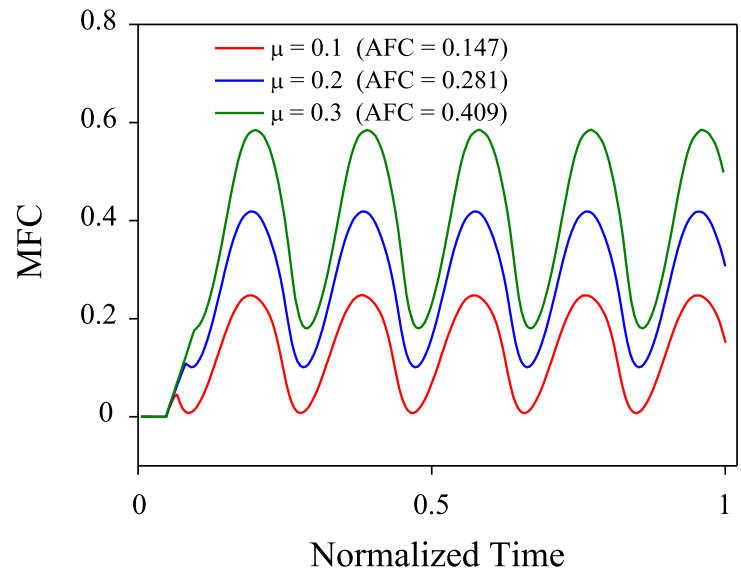

(d) MFC in setup (b) for varying $\mu$

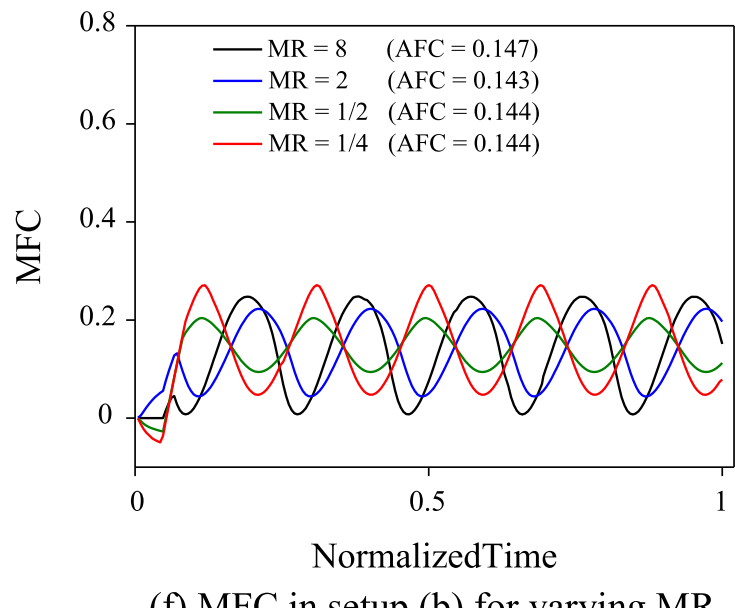

(f) MFC in setup (b) for varying MR

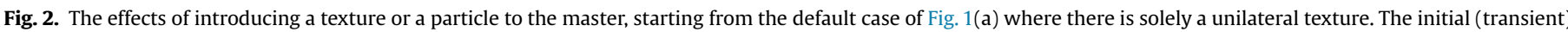

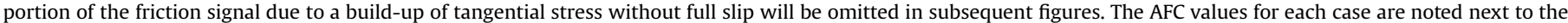
corresponding legend entry. 
again on soft interface friction. In particular, bulk heterogeneities embedded within the master would have a negligible effect on the macroscopic friction at small deformations, which will be observed within the numerical investigations. It should also be noted that, even if all microscopic variables were fixed, variations in the load distribution or refinements in the geometrical details on the mesoscale can also influence the measured macroscopic friction coefficient [41,42]. Presently, only a single texture type is considered and the macroscopic pressure is applied always uniformly.

In the case of a composite master, the MFC signal generated will additionally be highly oscillatory in time. Observations regarding the influences of the bulk and surface heterogeneities will be based upon the analysis of these oscillations. Specifically, the MFC signal will be characterized through its time average (AFC) and peak-to-peak oscillation magnitude $\left(\mathrm{P}_{\text {to }} \mathrm{P}-\mathrm{MFC}\right)$. When necessary, the variation of MFC will be plotted with respect to normalized simulation time that indicates the extent to which the master has completed three passes over the slave, which is sufficient for the moving time average of AFC to converge.

\section{Bulk heterogeneities versus surface heterogeneities}

Having introduced the structure of the default computational setup and the relevant parameters, initial analyses will compare the independent influences of introducing a texture or a particle to the master. Identifying the individual influences on the MFC signal will gain importance in later stages while constructing a method to tune the MFC signal. For this purpose, two computational setups were prepared: one with a bilateral texture and the other with a composite master - see Fig. 2(a,b). In the case of a bilateral texture, where the master is also assigned a sinusoidal surface texture, MFC undergoes significant oscillations due to the commensurate periodicity on both surfaces. Such oscillations are primarily responsible for the behavior that is reminiscent of stick-slip at the macroscale. On the other hand, it is observed that the MFC signal manifests similar oscillations when a stiff particle is embedded in the master, despite the fact that the interface is only unilaterally textured, because the texture features on the slave can interact significantly with the particle in the master due to finite deformations. Therefore, oscillations observed in the macroscopic friction signal can be either due to (i) one micro-textured surface sliding over another micro-textured surface, or (ii) a microscopically flat surface with subsurface inclusions sliding over a micro-textured surface. The similar responses from these two entirely different setups are further addressed by comparing them when the microscopic friction coefficient $\mu$ is varied. In both of the computational setups, an explicit rise in the mean of the MFC signal is observed when $\mu$ is increased (Fig. 2(c,d)). Hence, a sinusoidal asperity or a particle imposed on an initially unilaterally textured interface not only induces similar oscillations in the macroscopic response but their mean values also show similar trends when a major microscopic control parameter is varied.

For the bilaterally textured setup, the oscillation magnitude of the MFC signal is primarily controlled through $\mathrm{RMS}_{\mathrm{r}}$, the influence of which is demonstrated in Fig. 2(e). On the other hand, for the setup with a composite master, the oscillation magnitude can similarly be modified by varying the relative stiffness of the embedded particles, as demonstrated in Fig. 2(f). Two important statements may be made concerning these results. First, the mean of the friction signal (AFC) does not change appreciably in either case. Second, it is observed that the phase shift of the generated MFC signal is determined to a large degree by whether the particle is stiffer or softer than the base master material. In particular, the oscillations in the cases where the particles are twice or half as stiff are almost completely out of phase.
Overall, it is clear that the bulk and surface heterogeneities can induce macroscopically indistinguishable influences despite the entirely different microscopic mechanisms which underlie the observed macroscopic responses. This observation will be instrumental in tuning the macroscopic response by blending these two types of heterogeneities.

\section{Phase of the friction signal}

Individual influences of surface and bulk heterogeneities on the macroscopic response and the parallelism observed in them provide initial motives in constructing a methodology for tuning an oscillatory MFC signal in the presence of a bilaterally textured interface. Since the magnitude of the oscillation can relatively easily be controlled for both surface and bulk heterogeneities, the key factor in a successful tuning approach will be the ability to control the phase of the signal. For the case of a bilateral texture, the phase is simply shifted in direct correlation with the initial relative positions of the peaks of the two surfaces. This effect will therefore be eliminated by always initiating motion from the position of matching peaks in all subsequent simulations. For the case of a composite master, the investigations of Fig. 2 (f) essentially indicate that the phase of the MFC signal is controlled by the positioning of alternating regions of soft and stiff material within the master, which is determined by the parameter MR for a fixed particle position. Note that, in comparison to the phase in a bilateral texture, the variation of the phase with MR is comparatively more complex since it is accompanied by a change in $\mathrm{P}_{\mathrm{to}} \mathrm{P}-\mathrm{MFC}$.

To have a better perspective about the phases that an asperity and a particle manifest, MFC signals of three setups (with a bilateral texture, with a stiff particle and with a soft particle) are compared in Fig. 3(a). Here, it can clearly be observed that the soft particle behaves in an opposite manner not only to the stiff particle but also to the surface asperities in terms of the MFC signals. The former leads to a local reduction in the tangential contact stiffness whereas the latter two effectively increase this local stiffness. Hence, the oscillations due to the stiff particle and the asperity are almost in phase, with a small phase shift of $\lambda_{0}$. It is possible to further minimize $\lambda_{0}$ by varying the position of the embedded particle, since the phase shift depends on the distribution of soft and stiff regions within the master. This also means that the phase shifts $\lambda_{1}$ and $\lambda_{2}$ are controllable parameters.

Phase characteristics of the MFC signals for the three setups can also be monitored visually. For this purpose, simulation instances at the moments of maximum and minimum MFC signals are provided in Fig. 3(b,c). The bilaterally textured setup has its minimum MFC value when the asperities of the master just begin to slide down the asperities of the slave, whereas the maximum is reached when the asperities of the master are just passing the valleys of the slave. In the case of a composite master, the times of the peaks change based on the stiffness of the embedded particle. For instance, if the particle has a larger stiffness than the master matrix (MR $>1)$, oscillation maxima occur as the particle climbs up the asperity of the slave while minima occur as the particle slides down. Overall, (i) when softer regions meet the asperities of slave MFC reaches its maximum value, and (ii) when stiffer regions meet the asperities MFC reaches its minimum value. Note that this trend is opposite to the variation in the local normal contact stiffness, which is maximum when stiffer regions meet the asperities.

The observations stated so far suggest that a microscopic surface heterogeneity or a microscopic bulk heterogeneity can generate, through proper choices of $\mathrm{RMS}_{\mathrm{r}}$ and MR, MFC signals of matching $\mathrm{P}_{\text {to }} \mathrm{P}-\mathrm{MFC}$ but possibly non-matching phases. It has 


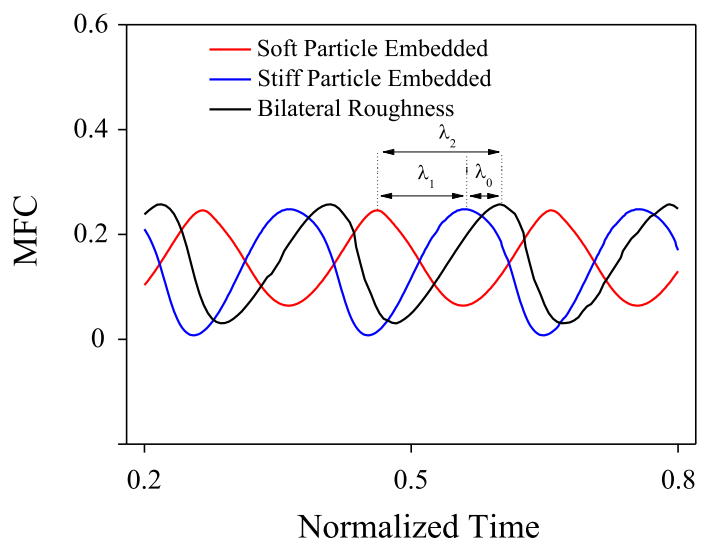

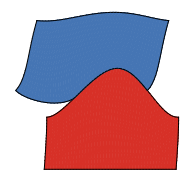

(b) Max: Asperity

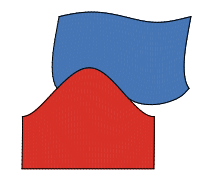

(c) Min: Asperity

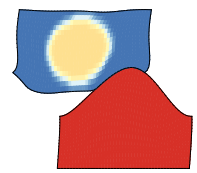

Stiff Particle

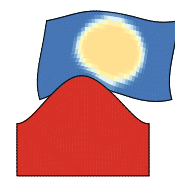

Stiff Particle

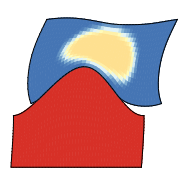

Soft Particle

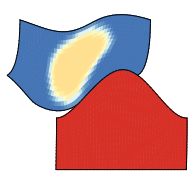

Soft Particle

(a) MFC of three cases

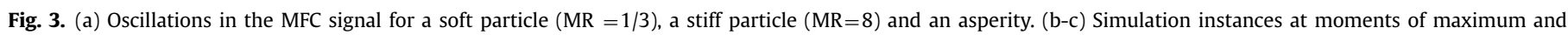
minimum MFC. See Fig. 2(a,b) for the starting configurations for these setups.

already been observed that AFC is not altered appreciably in either heterogeneity case with respect to the default unilateral texture setup where oscillations are not present. Therefore, when both types of heterogeneities are simultaneously imposed onto the master, the blended MFC signal will reflect whether each heterogeneity type reinforces or attenuates the influence of the other according to how well their phases match or mismatch. In particular, it is conceivable that there exist optimally (mis)matching bulk and surface heterogeneities such that the $\mathrm{P}_{\text {to }} \mathrm{P}-\mathrm{MFC}$ value significantly increases or decreases. The latter possibility may be interpreted as the presence of a bilateral micro-texture being shielded from macroscopic detection through the presence of the subsurface particles. Such possibilities for tuning the macroscopic friction signal are quantitatively investigated next.

\section{Tuning the oscillations of the friction signal}

In order to quantify the interaction between surface and bulk heterogeneities, the macroscopic friction signal was computed for a particle with a given stiffness that is placed at different positions across the master unit-cell in the presence of a bilateral texture. Specifically, MR was varied from 8 to $1 / 4$ and the horizontal axis was sampled at 18 unit-cell coordinates. Subsequently, $\mathrm{P}_{\text {to }} \mathrm{P}-\mathrm{MFC}$ was monitored in order to detect significant changes in the oscillation magnitude at specific particle stiffness-position combinations.

Fig. 4(a) displays the view of the setup with the particle at different positions, whereas Fig. 4(b) demonstrates the view of the setup at the same moment during sliding, with particles that have different stiffness values, where one can visually assess the increasing severity of deformation that the master undergoes when the particle embedded in it becomes softer. Overall, three interesting observations may be made. First, for very soft particles
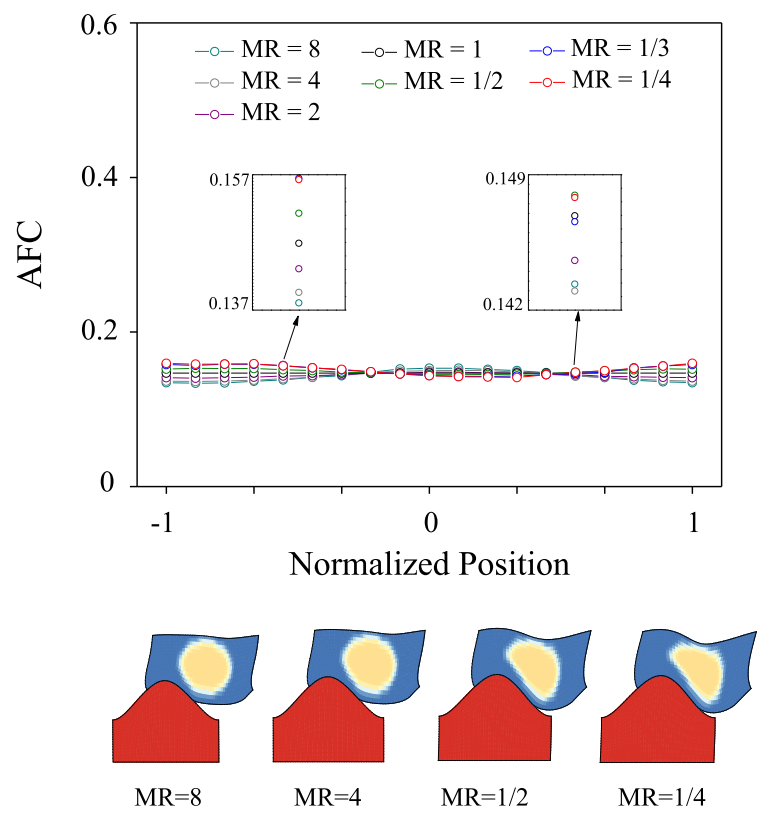

(b) AFC for varying MR and position

(a) $\mathrm{P}_{\text {to }} \mathrm{P}-\mathrm{MFC}$ for varying $\mathrm{MR}$ and position

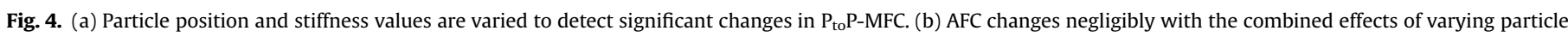

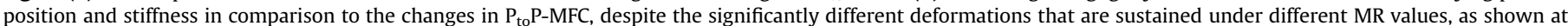
the same instance of sliding. 
( $M R=1 / 4)$, there clearly exists an optimal unit-cell position (slightly to the left of the peaks) at which $\mathrm{P}_{\text {to }} \mathrm{P}-\mathrm{MFC}$ decreases significantly down to 0.04 from a value of more than 0.2 , the one obtained in the absence of the particle. At such small values, the influence of the bilateral texture on the macroscopic interface is expected to be significantly less pronounced. As anticipated based on preceding discussions, because soft particles placed at this optimal point are able to attenuate the oscillatory behavior generated due to the presence of the asperity, stiff particles placed at the same optimal point reinforce the oscillations. In particular, this reinforcement is also optimal, i.e. a maximum $\mathrm{P}_{\text {to }} \mathrm{P}-\mathrm{MFC}$ is obtained. Hence, the optimal location appears to be an extremum for any choice of stiffness value.

The second observation is that the role of soft and stiff particles is reversed if the particle is moved significantly to the right of the peaks. In this case, even stiff particles can attenuate the oscillations while soft particles reinforce them. Clearly, the optimal locations would also be reversed if the sliding direction is reversed (cf. Fig. 4(a)). Third, in comparison to the observed changes in $\mathrm{P}_{\text {to }} \mathrm{P}-$ MFC, AFC values do not vary significantly with changing particle stiffness, which was observed earlier as well. Changes in AFC are especially small in magnitude when the particle is positioned near the asperity of the master. Hence, the oscillations in the MFC signal are roughly around the same mean, which is in most of the cases considerably close to 0.143 - the AFC value that was obtained in the default setting (Fig. 1(a)). This is particularly important because it demonstrates an ability to influence the magnitude of the oscillations alone, without altering the mean value. The mean value itself is essentially controlled by independent mechanisms such as the macroscopic contact pressure or the microscopic friction coefficient.
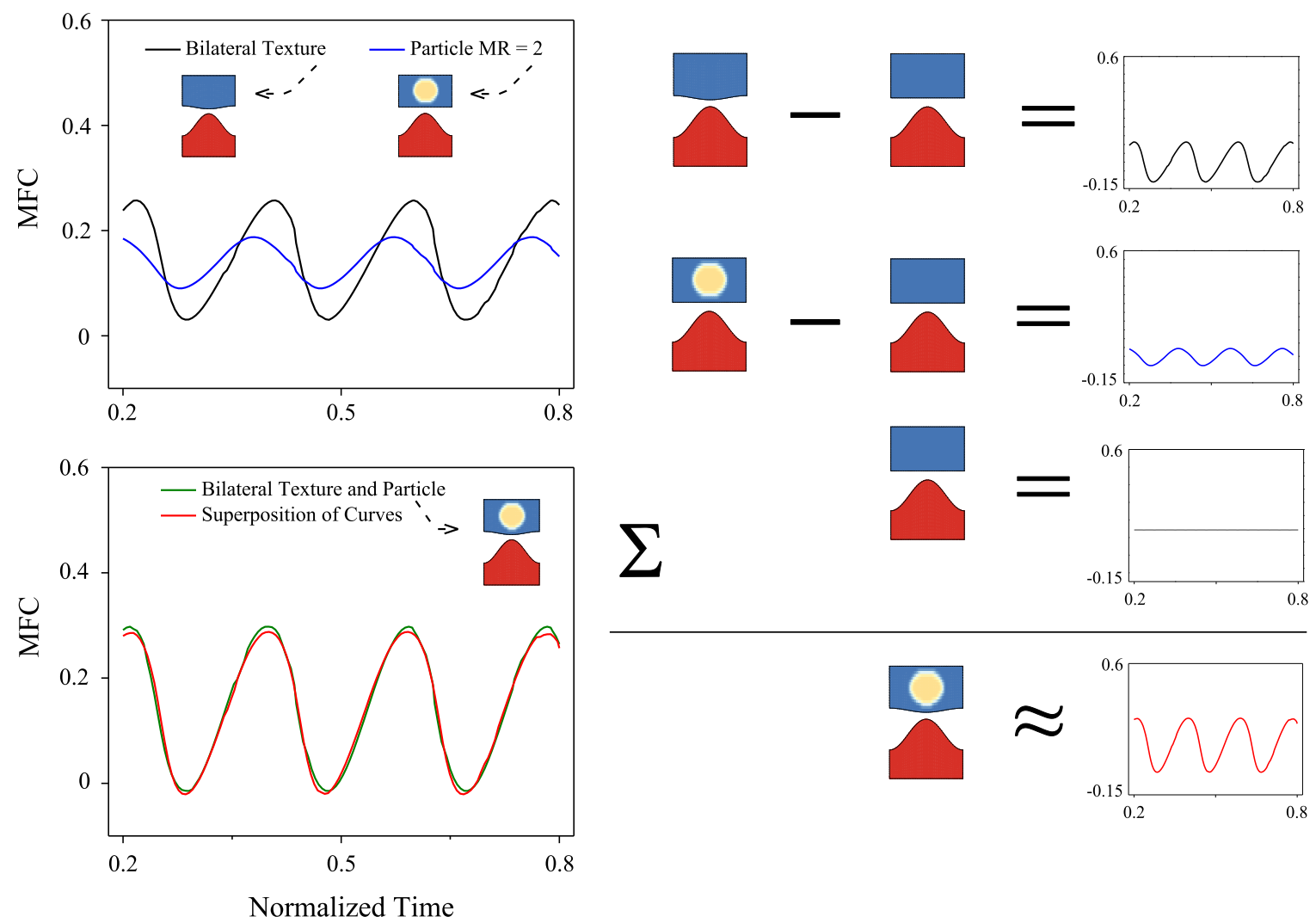

$\Sigma$
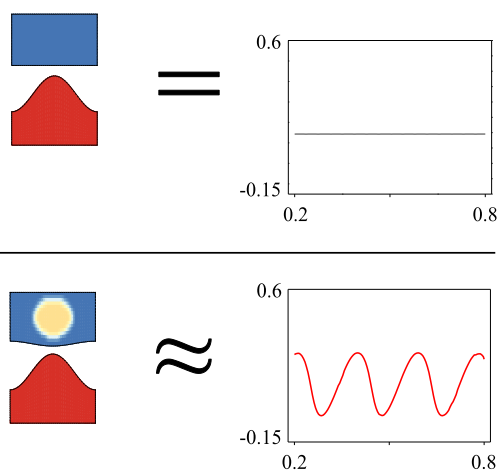

0.8

\section{Superposition of decoupled friction signals}

Identifying optimal particle locations in order to minimize or maximize the oscillations requires multiple computations as the particle is scanned across the master unit-cell. An analytical tions would or generating initial guesses for the optimal locaeffort and help clarifying the mechanism of reinforcement and of the macroscopic friction (AFC) does not vary considerably due to the individual or simultaneous imposition of surface and bulk heterogeneities onto the master. It is thus tempting to examine the oscillatory friction signal of varmonstrates the underlying operations for analyzing the MFC signal along this idea for the setup with a bilaterally textured topography in the absence of particles and for the setup with a unilateral texture in the presence of a particle. In a first step, the MFC signal is decomposed as

$\mathrm{f}(\mathrm{t})=\overline{\mathrm{f}}(\mathrm{t})+\tilde{\mathrm{f}}(\mathrm{t})$

Here, $\bar{f}(t)$ represents the AFC value from the unilateral texture alone, approximately corresponding to the mean of $\mathrm{f}(\mathrm{t})$ in all cases, and $\tilde{\mathrm{f}}(\mathrm{t})$ is the remaining plain oscillatory signal, i.e. it has approximately zero mean. For the bilaterally textured case, $\tilde{f}(t)$ asperity A similar interpretation holds for the case of a composite presence in the master. Taking this decomposition one signals associated with asperity and particle influences through simple superposition to obtain
Normalized Time

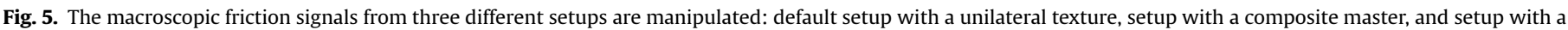
bilateral texture. The approximate curve obtained through superposition is then compared with the actual computational outcome. 
$\tilde{\mathrm{f}}(\mathrm{t})_{\text {combined }}=\tilde{\mathrm{f}}(\mathrm{t})_{\text {asperity }}+\tilde{\mathrm{f}}(\mathrm{t})_{\text {particle }}$.

In this manner, a new plain oscillatory signal may be obtained which is indicative of the combined influence on the default setup when asperities and particles are simultaneously imposed on the master. In order to obtain the overall friction signal, the final step is to add the common mean value to this combined response:

$\mathrm{f}_{\text {approx }}(\mathrm{t})=\overline{\mathrm{f}}(\mathrm{t})+\tilde{\mathrm{f}}(\mathrm{t})_{\text {combined }}$.

$\mathrm{f}_{\text {approx }}(\mathrm{t})$ now corresponds to an approximation which represents the prediction of the coupled MFC signal $f(t)$ from the setup that has bilateral texture together with a composite master. Clearly, the predictive capability of this superposition approach cannot be guaranteed since the addition of an asperity or a particle entirely alters the problem setup which itself is highly nonlinear due to contact interactions and finite deformations. Despite this potential theoretical shortcoming, Fig. 5 demonstrates that the overall oscillation curve that is produced from this superposition approach delivers a very good estimate of the actual coupled response for both soft and stiff particles. This predictive potential suggests that without preparing a computational setup which features a bilaterally textured topography and a composite master, one is able to estimate the oscillatory MFC signal generated due to the combination of a bilateral texture and a particle at any position simply by examining the individual MFC signals of the two independent cases. Fig. 6(a,b) further demonstrates the superposition approach

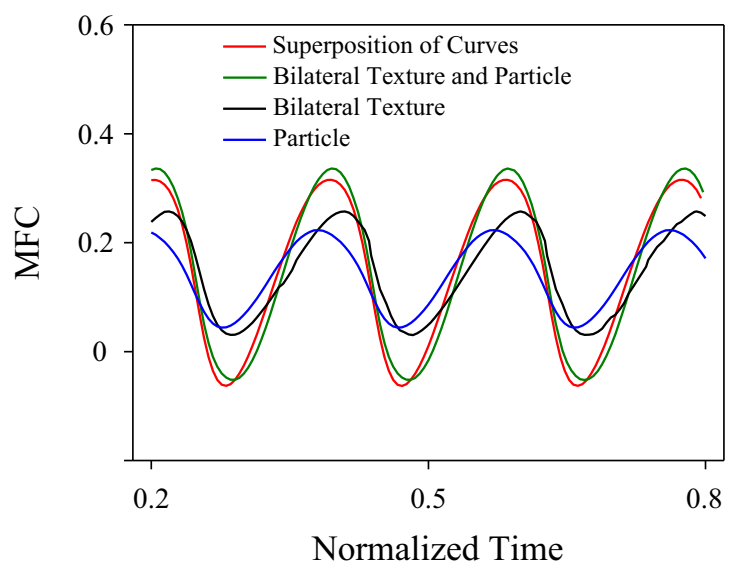

(a) $\mathrm{MFC}$ prediction for $\mathrm{MR}=4$

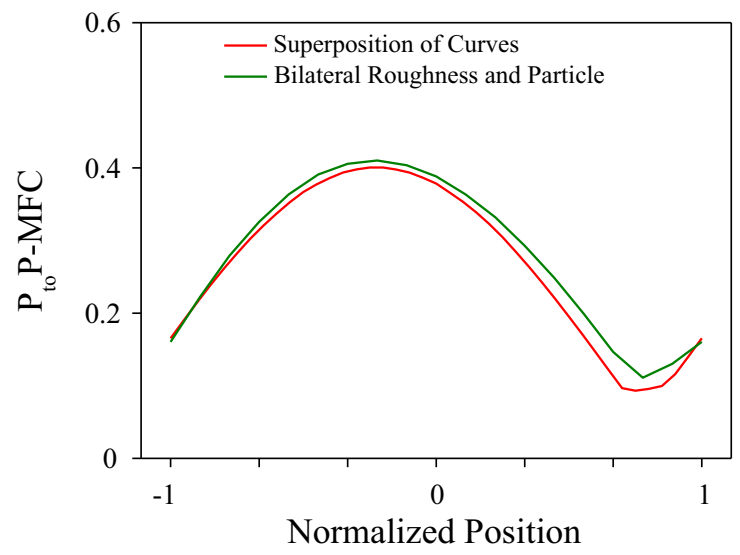

(c) $\mathrm{P}_{\text {to }} \mathrm{P}-\mathrm{MFC}$ prediction for $\mathrm{MR}=4$ for two additional scenarios with stiffer and softer particles. The agreement between the MFC curves obtained computationally and based on the superposition approach is not perfect due to the lack of a solid theoretical foundation for superposition. Nevertheless, the actual computational outcome is predicted with good accuracy in both cases. Overall, these results are in agreement with the initial observations stated in Section 4 which motivated the feasibility for tuning the macroscopic friction signal.

The predictive capability of the superposition approach is further demonstrated in Fig. $6(\mathrm{c}, \mathrm{d})$ where $\mathrm{P}_{\mathrm{to}} \mathrm{P}-\mathrm{MFC}$ is monitored as the primary quantity of interest with varying particle position. Here, the original curves for stiff and soft particles correspond to those in Fig. 4(a) while the superposition curves are simply generated by shifting the $\tilde{f}(t)$ particle signal in time before calculating $\mathrm{f}_{\text {approx }}(\mathrm{t})$ via $\tilde{\mathrm{f}}(\mathrm{t})_{\text {combined. }}$. Specifically, denoting the signal oscillation period with $\mathrm{T}$ (see Table 1 ), if the particle is shifted by $\alpha \in[-1,1]$ on the normalized position axis then one simply employs

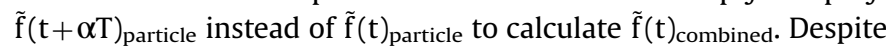
the very good agreement with the original curves that are obtained via direct micromechanical simulations, the superposition approach will not be employed further in this work.

\section{Influence of major numerical parameters}

Having established, partly validated and understood the manner in which MFC from a bilateral texture can be tuned by

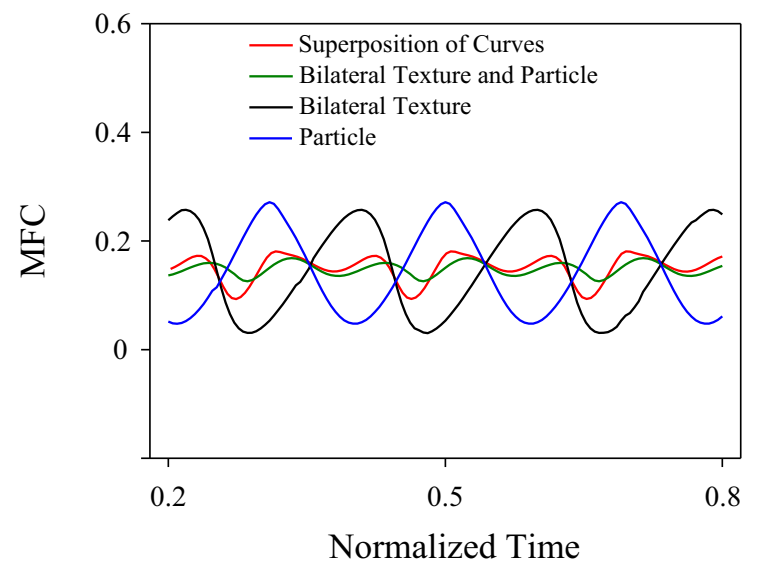

(b) $\mathrm{MFC}$ prediction for MR $=1 / 4$

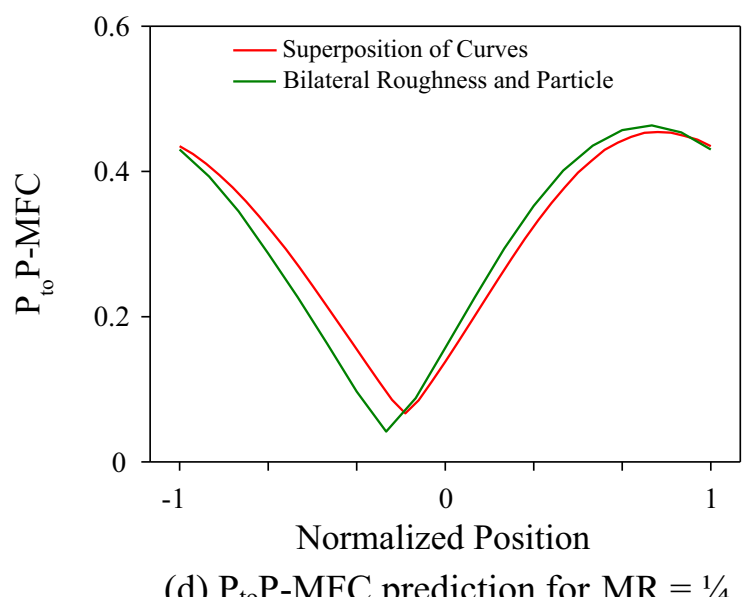

(d) $\mathrm{P}_{\text {to }} \mathrm{P}-\mathrm{MFC}$ prediction for $\mathrm{MR}=1 / 4$

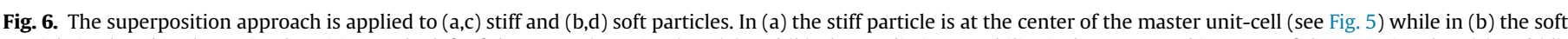

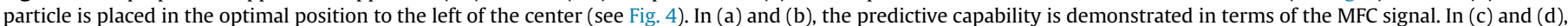
this capability is demonstrated directly in terms of the oscillation magnitude as the particle position is varied. 


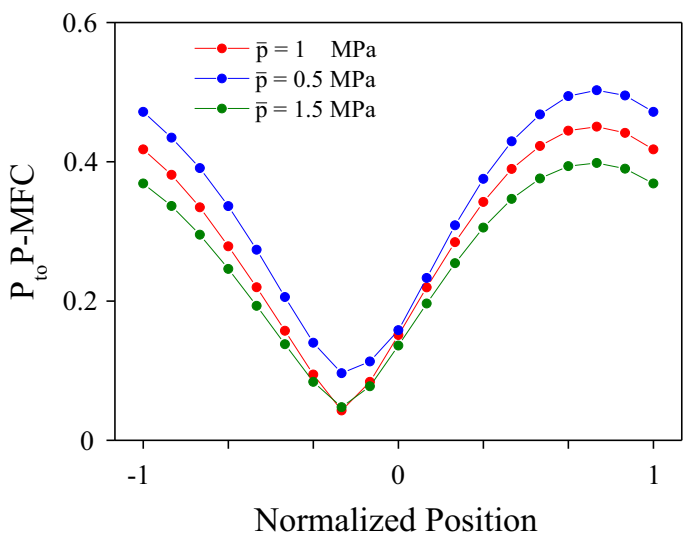

(a) $\mathrm{P}_{\text {to }} \mathrm{P}-\mathrm{MFC}$ for varying $\overline{\mathrm{p}}$

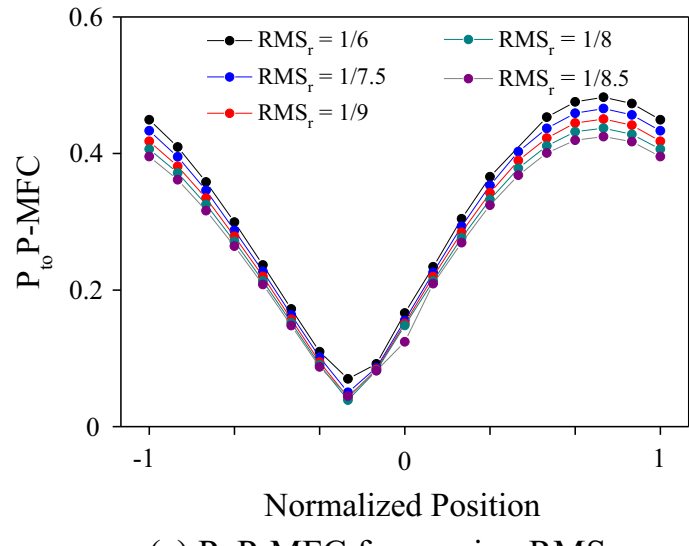

(c) $\mathrm{P}_{\text {to }} \mathrm{P}-\mathrm{MFC}$ for varying $\mathrm{RMS}_{\mathrm{r}}$.

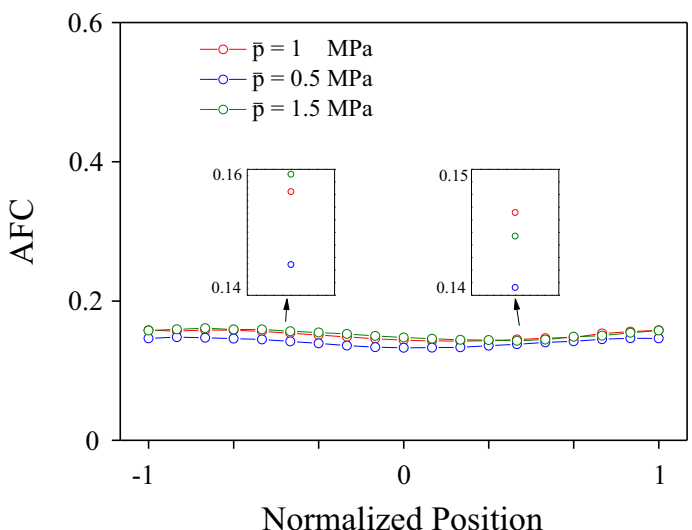

(b) AFC for varying $\bar{p}$

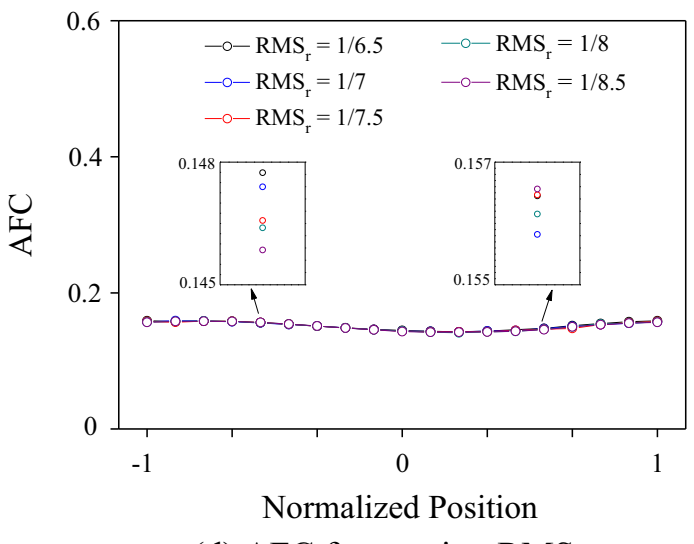

(d) AFC for varying $\mathrm{RMS}_{\mathrm{r}}$.

Fig. 7. Macroscopic pressure $(\overline{\mathrm{p}})$ and texture amplitude $\left(\mathrm{RMS}_{\mathrm{r}}\right)$ are varied. In both cases, a soft particle has been employed with $\mathrm{MR}=1 / 4$.

embedding particles into the master, the influence of major numerical parameters will be investigated next. The results which have been presented so far have been mostly based on the default parameter set, except for variations which have been explicitly noted such as those regarding the particle position and MR. In order to concentrate on the detection of shifts in optimal particle locations, changes in $\mathrm{P}_{\text {to }} \mathrm{P}-\mathrm{MFC}$ extrema and possible influences to AFC, the remaining investigations will solely be based on a bilateral surface texture with a composite master.

The influence of the surface texture can either be varied from the outset by changing the texture amplitude via $\mathrm{RMS}_{\mathrm{r}}$ or by changing the macroscopic contact pressure via p. Fig. 7 summarizes the effect of these two parameters as the particle scans the master unit-cell. Specifically, Fig. 7(a) demonstrates that a decrease in $\bar{p}$ reduces the particle influence in view of an increasing $\mathrm{P}_{\mathrm{to}} \mathrm{P}-\mathrm{MFC}$ since, as remarked in Section 2, not only the macroscopic pressure-dependence of AFC (Fig. 7(b)) but also the particle influence are both strictly finite deformation effects. On the other hand, $\mathrm{RMS}_{\mathrm{r}}$ variations again do not influence AFC but only alter $\mathrm{P}_{\text {to }} \mathrm{P}-\mathrm{MFC}$ (Fig. $7(\mathrm{c}, \mathrm{d})$ ). In either case, the optimal position is observed to remain unchanged.

The material model employed as well as the size of the particle may also be varied. Fig. 8(a) demonstrates that the size of the particle, controlled via VF, can significantly alter $\mathrm{P}_{\text {to }} \mathrm{P}-\mathrm{MFC}$. On the other hand, switching the default material model employed in the simulations for all the finite elements in the mesh from Ogdentype to Neo-Hookean while preserving the bulk and shear moduli leads to only minor changes despite the significantly different hyperelastic responses (Fig. 8(c)). In either case, the optimal particle locations and AFC do not change appreciably in comparison to the changes which are observed in $\mathrm{P}_{\text {to }} \mathrm{P}$-MFC (Fig. 8(b,d)).

Among all the parameters investigated, the microscopic friction coefficient $(\mu)$ is the one which has a noticeably large influence on the optimal particle position (Fig. 9). As $\mu$ is increased from 0 to 0.3 , optimal particle position is shifted towards the left. This shift correlates with the fact that the peaks on the master also get deformed towards the left during frictional sliding to the right. Indeed, in the case of $\mu=0$ the average shift in the peaks of the master due to deformation is zero and the optimal position is also the center of the unit-cell.

It is remarked that the deformation of the contact interface significantly increases with increasing $\mu$. This leads not only to an increasing AFC but also to an increasing $\mathrm{P}_{\text {to }} \mathrm{P}-\mathrm{MFC}$, since the latter is an indication of the strain energy which is accumulated and subsequently released during macroscopic sliding of the interface. The accumulation of the strain energy is gradual although its release is relatively rapid. This leads to a rapid change in the MFC signal within a very narrow period of time, almost like a jump, which is challenging to capture in the default quasi-static simulation setting that is employed in this work. The data points in Fig. 9 which lie between dotted lines indicate those computations for which this quasi-static setting did not deliver convergence. For these points alone, computations were carried out by incorporating inertia but with a density that was scaled down to the range $10^{-5}-10^{-7} \mathrm{~kg} / \mathrm{m}^{3}$ in order to ensure that the influence of inertia is indeed negligibly small. At such small values, inertia is not physical but simply serves to regularize the sudden jumps in the MFC signal by inducing a small slope, thereby avoiding the convergence problem without affecting the macroscopic predictions. 


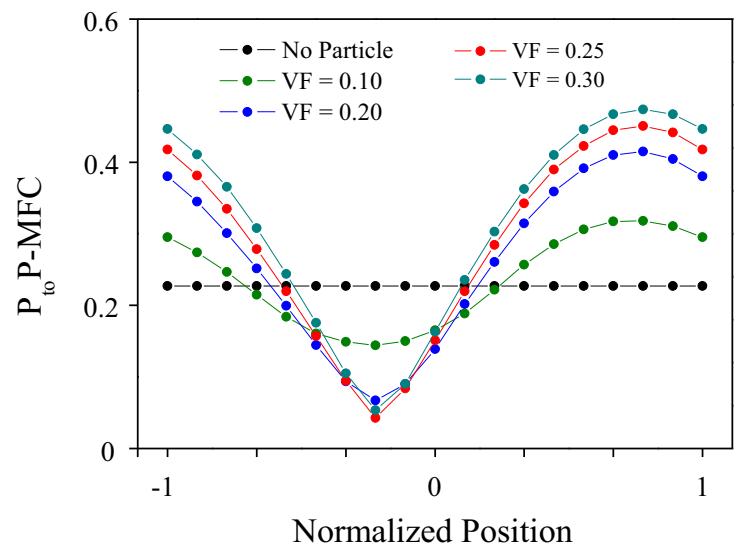

(a) $\mathrm{P}_{\text {to }} \mathrm{P}$-MFC for varying $\mathrm{VF}$

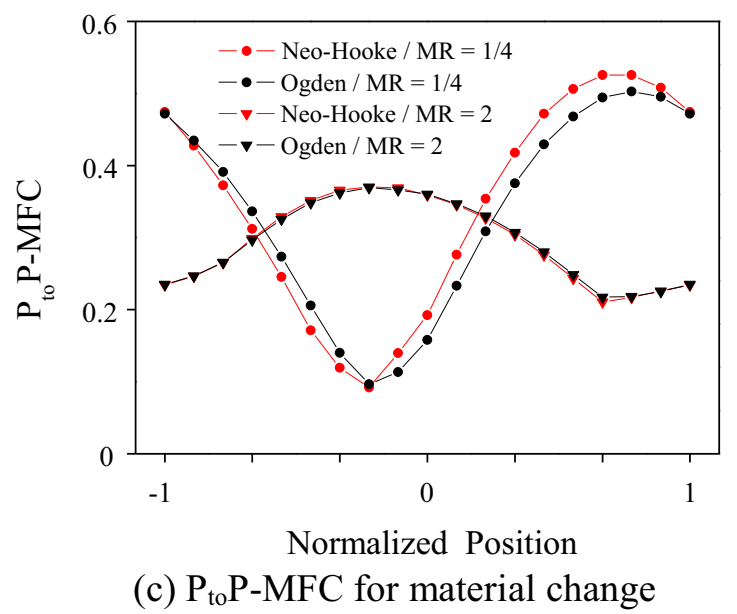

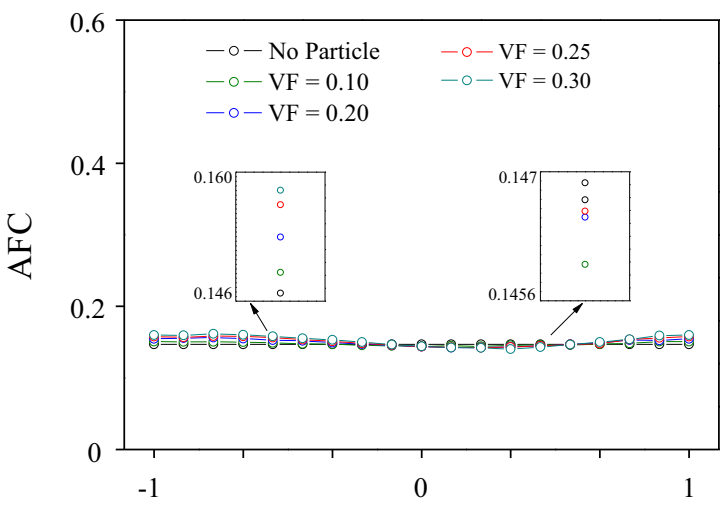

Normalized Position

(b) AFC for varying VF

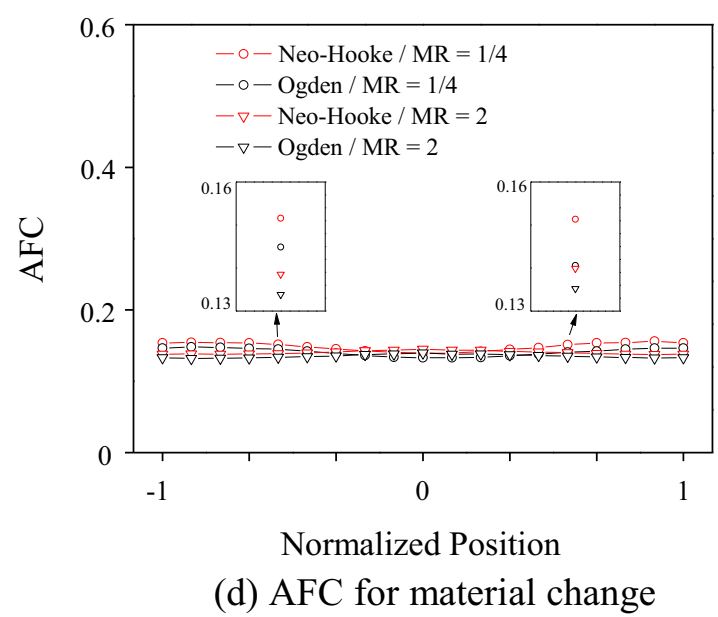

Fig. 8. VF and material model are varied: (a,b) employ soft particles with $M R=1 / 4,(c, d)$ employ stiff $(M R=2)$ and soft $(M R=1 / 4)$ particles.

\section{Limit responses}

MFC and, therefore, AFC or $\mathrm{P}_{\text {to }} \mathrm{P}-\mathrm{MFC}$ approach limit values as specific numerical parameters are varied. Among these, the vertical position of the particle in the master determines the degree to which the particle can interact with the surface texture. Since the default height of the master unit-cell is $6 \mu \mathrm{m}$ and the particle is already very close to the top border of the unit-cell, the height was increased to $9 \mu \mathrm{m}$ to gain vertical space for particle position variation. In order to preserve the mesh resolution, the number of elements along this direction was also increased. Vertical scanning was carried out using both stiff and soft particles, with MR equal to 2 and $1 / 4$ respectively. Fig. 10 essentially demonstrates that with increasing distance of the particle from the contact interface (measured with respect to its default position) the macroscopic friction signal converges to that of a bilaterally textured case without any particle embedded in the master, i.e. the influence of the particle diminishes. As in most cases, there is no appreciable change in AFC values, despite the significant changes in $\mathrm{P}_{\text {to }} \mathrm{P}-\mathrm{MFC}$. At this point, it may be noted that embedding multiple particles along the vertical direction would lead to the stiffening of the master material as a whole. Although the $\mathrm{P}_{\text {to }} \mathrm{P}-\mathrm{MFC}$ value would then still be predominantly influenced by the particle that is closest to the interface, this overall stiffening would hinder the deformation of the master surface as a whole and thereby lead to a pronounced change in AFC. Since the aim of the present work is to tune the oscillation magnitude without altering the mean value, this scenario will not be pursued further.
Similarly, varying the width and height of the unit-cells for a fixed particle location delivers limit responses. The number of elements was varied accordingly in both cases, in order to preserve the mesh resolution. Computations were again carried out for stiff and soft particles of a given particle size. As the width of the unitcell increases, the volume fraction of the particle effectively decreases and hence the $\mathrm{P}_{\mathrm{to}} \mathrm{P}-\mathrm{MFC}$ values for the two types of particle approach each other (Fig. 11(a)) which together converge onto the response from a bilaterally texture surface without a particle. Furthermore, the gradually vanishing oscillations of these three curves at large unit-cell widths are associated with the fact that an increasing period of the texture essentially diminishes its effect such that the response from a non-textured interface is approached. Indeed, Fig. 11(b) demonstrates that AFC decreases towards the microscopic value of the friction coefficient, $\mu=0.1$.

Although the width of the unit-cell is a physical variable, the height is a free variable which should ideally be assigned a sufficiently large value beyond which it has no influence on the computational outcome. This is associated with the fact that, in all cases, the aim is to embed a single particle at a fixed position in the vicinity of the contact interface and artificial stiffness generated from a too-close upper boundary should strictly be avoided. Fig. 12(a,b) demonstrate the influence of the height as it is increased from its default value $(6 \mu \mathrm{m})$ towards $10 \mu \mathrm{m}$, in order to detect if there were significant variations in $\mathrm{P}_{\text {to }} \mathrm{P}-\mathrm{MFC}$ and AFC values. The observed minor variations are not significant for either quantity, indicating that the default choice which minimizes the computation cost is already satisfactory. 


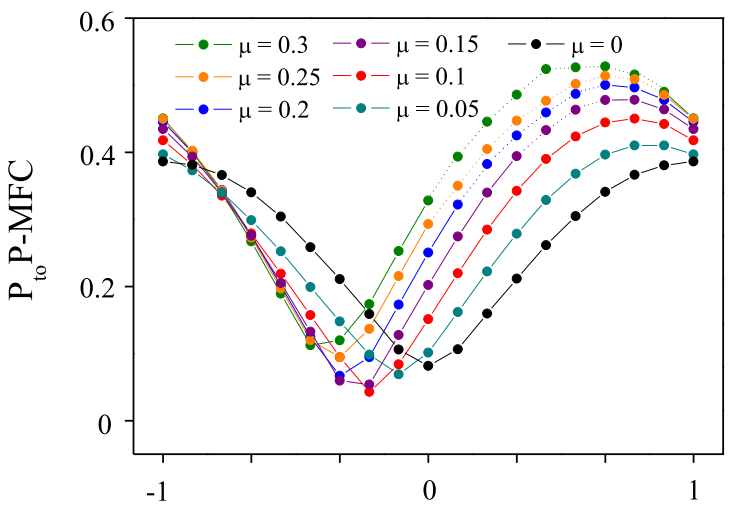

Normalized Position

(a) $\mathrm{P}_{\text {to }} \mathrm{P}-\mathrm{MFC}$ for varying $\mu$ with soft particle

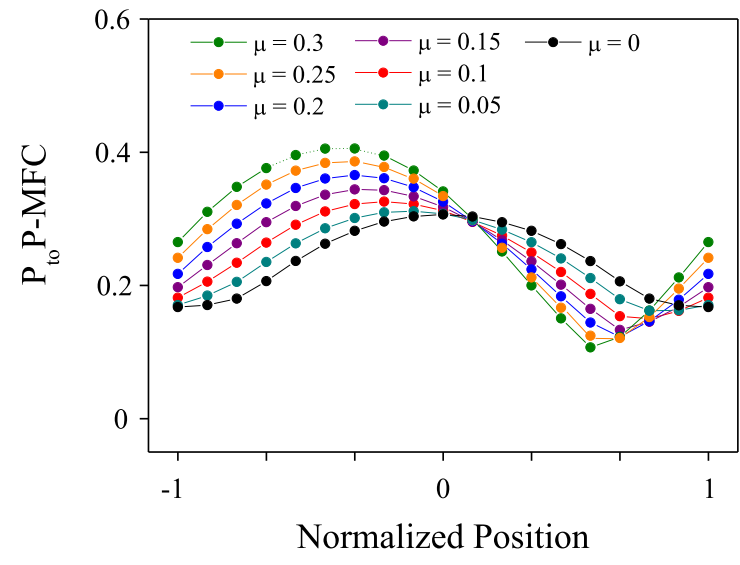

(c) $\mathrm{P}_{\text {to }} \mathrm{P}-\mathrm{MFC}$ for varying $\mu$ with stiff particle

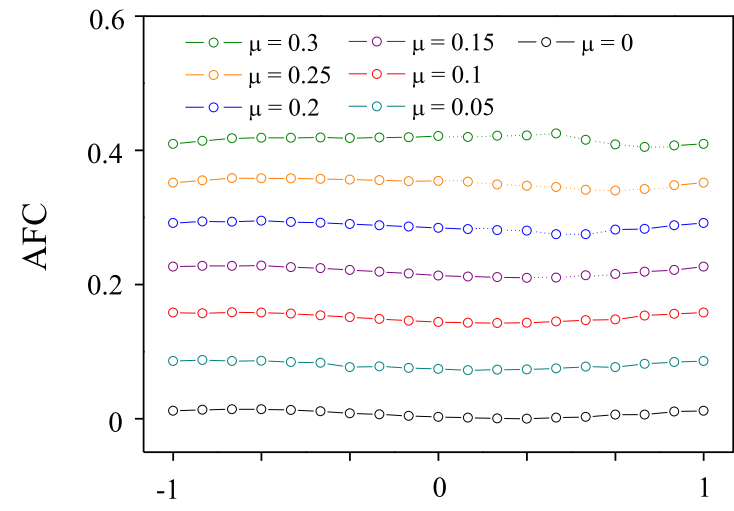

Normalized Position

(b) AFC for varying $\mu$ with soft particle

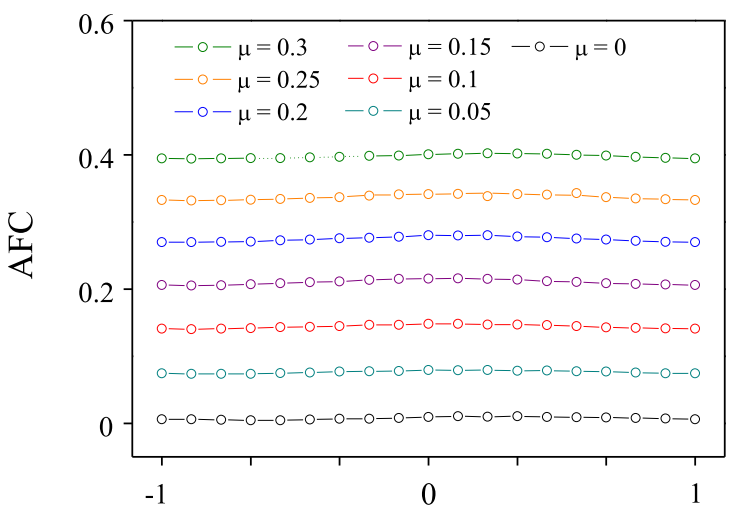

Normalized Position

(d) AFC for varying $\mu$ with stiff particle

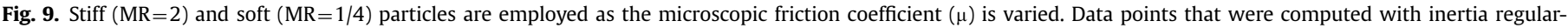
ization lie between dotted lines.

$G_{s}$ was also modified to observe the limit behavior of the setup (Fig. 13). For soft and stiff particles, $\mathrm{G}_{\mathrm{s}}$ was varied from $0.5 \mathrm{MPa}$ to 100MPa. Clearly, increasing values approach the limit where the slave surface is rigid. The significant variation in the macroscopic friction response beyond the default value (5MPa) also indicates that the slave surface was already significantly stiffer than the master in all the cases tested so far, yet it was far below a stiffness value where it could be approximated as a rigid surface. Hence, despite its default high stiffness, it is clearly important to capture the deformations that it sustains. On the other side of the spectrum, note that decreasing $G_{s}$ values do not deliver a limit response since this essentially renders the master stiffer in comparison to the slave. Even when the master is effectively acting as a rigid indenter, further decreasing slave stiffness is equivalent to increasing the macroscopic contact pressure, thereby leading to larger deformations and hence to continuous variations in the macroscopic response.

Among all the limit responses, those associated with the stiffness ratio MR are the most challenging to capture and require a partial reconsideration of various numerical parameters to ensure the reliability of the observations. In the ranges of MR values tested and reported in Fig. 4, a monotonic variation of $\mathrm{P}_{\text {to }} \mathrm{P}-\mathrm{MFC}$ was observed at an optimal particle location associated with an extremum point. In order to probe the macroscopic response beyond the values of MR which were already reported, MR has been additionally varied in the range $10^{-4}$ to $10^{2}$, i.e. from one extreme where the particle essentially represents a void to another where the particle is effectively rigid. As for the analysis of Fig. 10, to avoid convergence problems at small MR values where the oscillation amplitude increases significantly, the regularizing effect of inertia was employed with a scaled density. Additionally, in order to avoid possibly misleading effects of excessive mesh deformation at small MR values, the macroscopic pressure was reduced from its default value of $1 \mathrm{MPa}$ to $0.1 \mathrm{MPa}$. Finally, based on the analysis of Fig. 12(a), the height of the sample was also increased from $6 \mu \mathrm{m}$ to $10 \mu \mathrm{m}$ in order to completely eliminate any influence of the sample height. With these modifications, Fig. 14 summarizes the variations of $\mathrm{P}_{\text {to }} \mathrm{P}-\mathrm{MFC}$ and AFC. The latter does not change appreciably and, hence, attention is focused on the oscillations. As the particle becomes comparatively stiffer, $\mathrm{P}_{\text {to }} \mathrm{P}-\mathrm{MFC}$ increases as in Fig. 4 then stabilizes at the rigid particle limit. Towards the other extreme, $\mathrm{P}_{\text {to }} \mathrm{P}-\mathrm{MFC}$ again initially behaves by decreasing in magnitude as in Fig. 4 although a reverse trend starts to become prominent with further reduction in MR towards the void limit. Evidently, there exists an optimal stiffness value for soft particles in addition to an optimal position. For the present example, this value is approximately $\mathrm{MR}=1 / 4$.

The reason for the reversal in the $\mathrm{P}_{\text {to }} \mathrm{P}-\mathrm{MFC}$ trend may be explained by the large deformations associated with very soft particles. If the deformations of the particle at the same simulation instance are compared as MR decreases (Fig. 14(c)), it appears as though the lower surface of the particle snaps through. Hence, as the particle stiffness decreases, the interface between the particle and the surrounding matrix material starts to deform to such an 


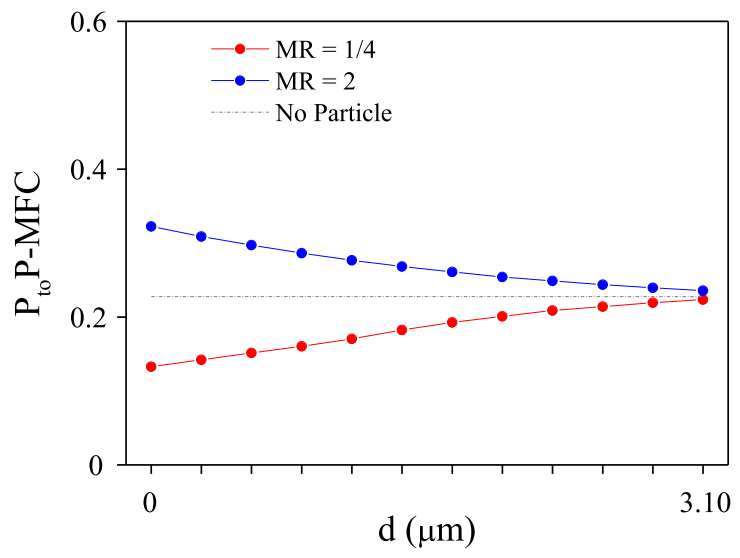

(a) $P_{\text {to }} P$ MFC for varying $d$
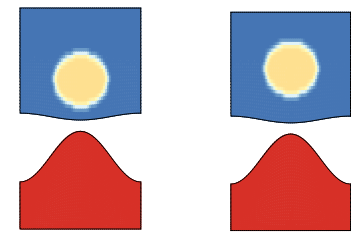

$\mathrm{d}=0 \mu \mathrm{m} \quad \mathrm{d}=1.13 \mu \mathrm{m}$

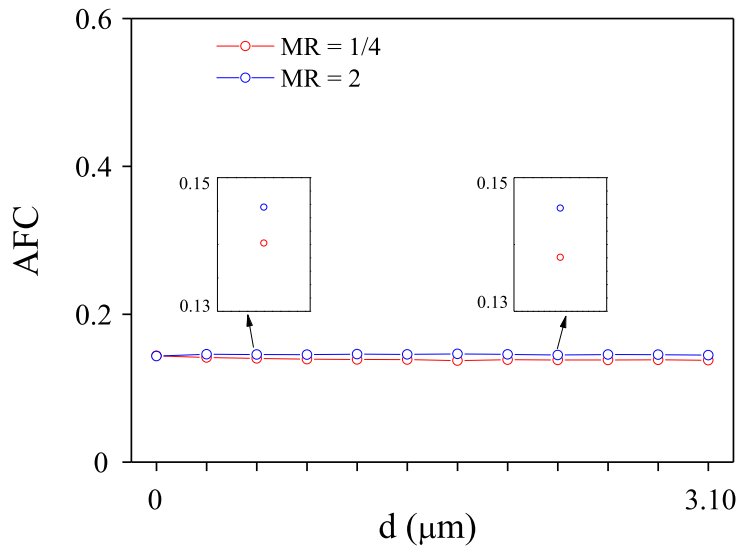

(b) AFC for varying $d$
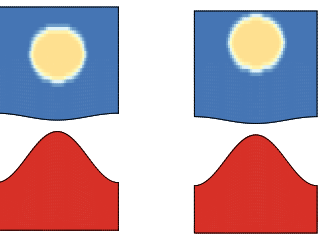

$\mathrm{d}=1.97 \mu \mathrm{m} \mathrm{d}=3.10 \mu \mathrm{m}$

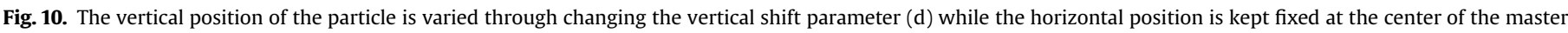

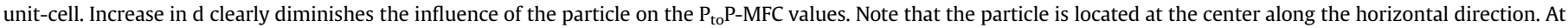
this location, the soft particle has an attenuating effect while the stiff particle has a reinforcing effect, which explains the different trends with increasing $\mathrm{d}$.

extent that two corners form where contact-like conditions locally start to prevail within the particle. These conditions are represented by rapidly increasing stresses that resist the approach of

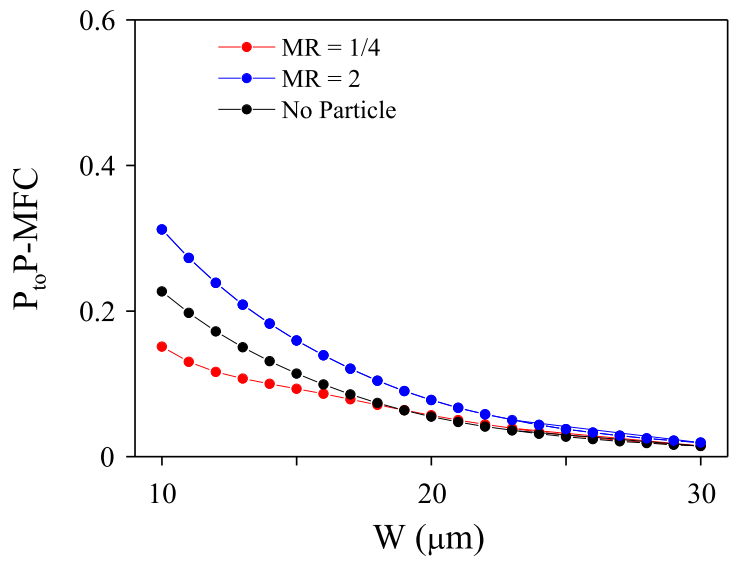

(a) $\mathrm{P}_{\text {to }} \mathrm{P}-\mathrm{MFC}$ for varying unit-cell width opposing points on the interface towards one another. In fact, since very small MR values represent a void, actual contact conditions would certainly be observed if the macroscopic pressure

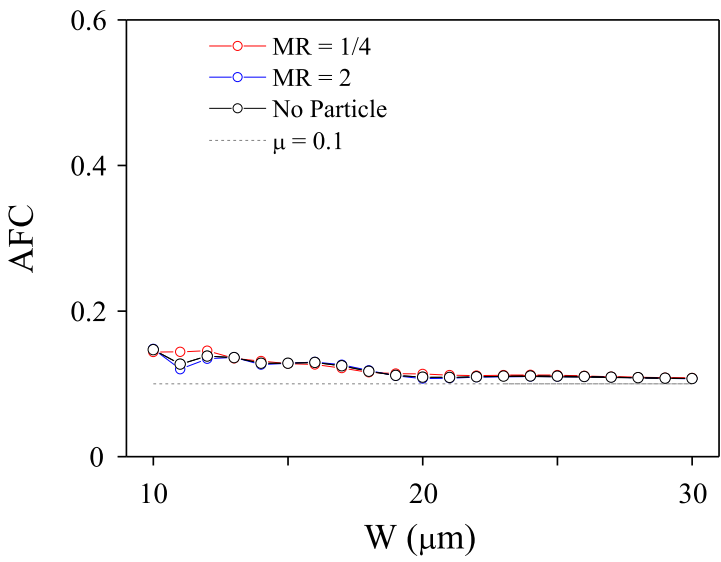

(b) AFC for varying unit-cell width
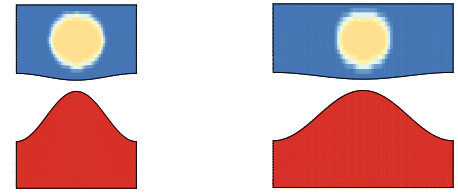

$\mathrm{W}=10 \mu \mathrm{m}$

$\mathrm{W}=15 \mu \mathrm{m}$

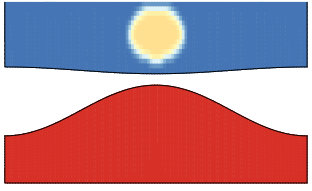

$\mathrm{W}=25 \mu \mathrm{m}$

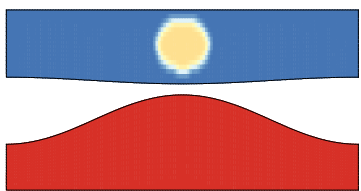

$\mathrm{W}=30 \mu \mathrm{m}$

(c) Starting configurations for the computation setups where the width (W) is increased

Fig. 11. Unit-cell size is varied by changing the width parameter $(\mathrm{W})$. Increasing $\mathrm{W}$ weakens the influence of both the particles and the texture. 


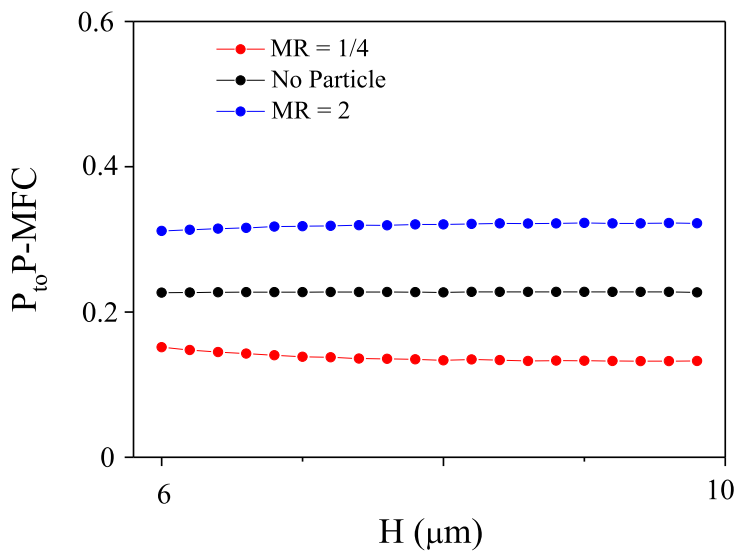

(a) $\mathrm{P}_{\text {to }} \mathrm{P}-\mathrm{MFC}$ for varying unit-cell height
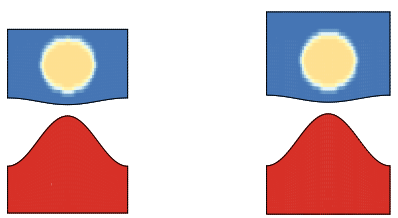

$$
\mathrm{H}=6 \mu \mathrm{m} \quad \mathrm{H}=7 \mu \mathrm{m}
$$

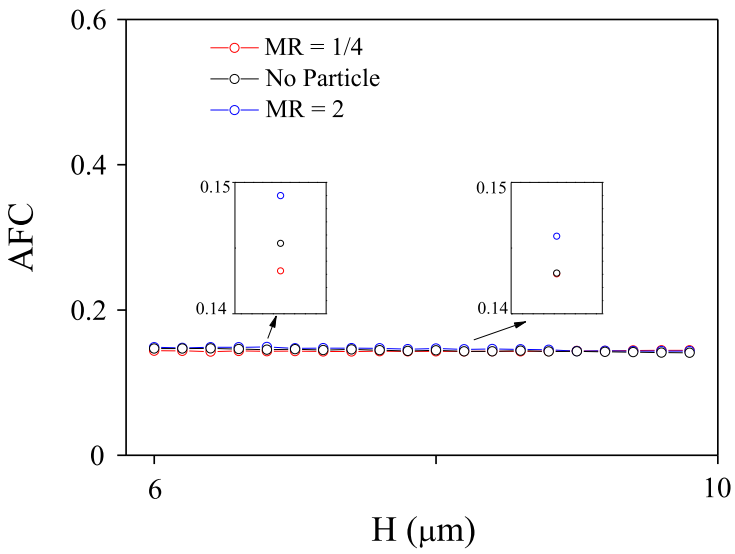

(b) AFC for varying unit-cell height
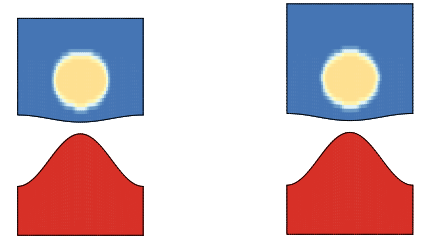

$\mathrm{H}=8 \mu \mathrm{m} \quad \mathrm{H}=9 \mu \mathrm{m}$

(c) Starting configurations for the computation setups where the height $(\mathrm{H})$ is increased

Fig. 12. Unit-cell size is varied by changing the height parameter $(H)$. Increasing $H$ does not have a significant influence on the macroscopic response.

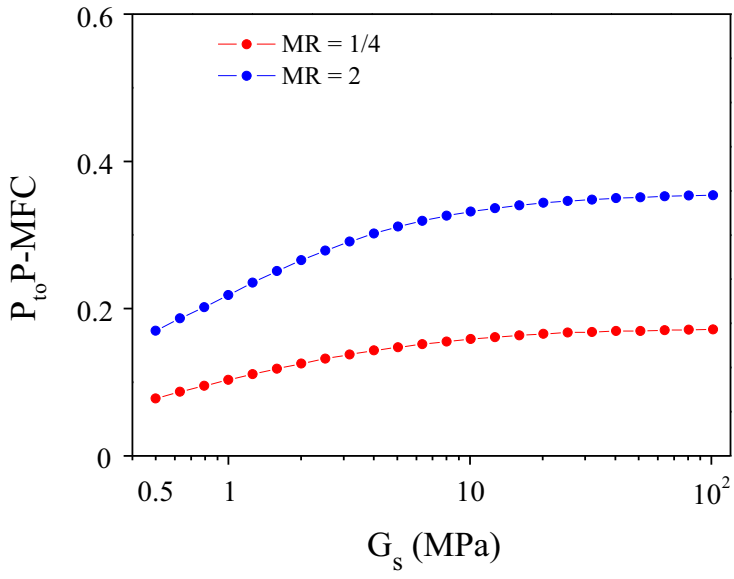

(a) $\mathrm{P}_{\text {to }} \mathrm{P}$ MFC for varying slave stiffness

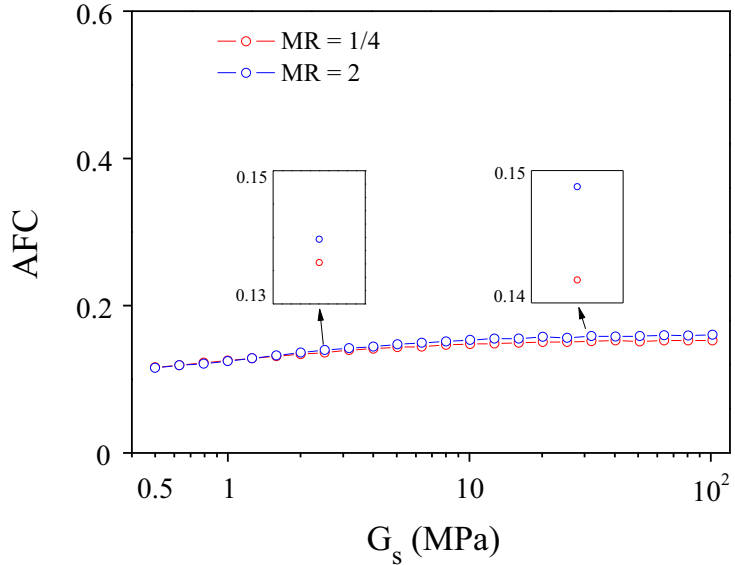

(b) AFC for varying slave stiffness

Fig. 13. Variation of the macroscopic response with the stiffness $\left(G_{s}\right)$ of the slave.

was increased further. Hence, it is clear that the mode of particle deformation does not remain the same at all MR values. This change in the microscopic deformation mode is responsible for the non-monotonic macroscopic response curve, which is not surprising in view of the highly nonlinear nature of the problem.

\section{Conclusion}

The macroscopically observed response of interfaces which are associated with soft materials is strongly governed by the interaction of the surface topography with the neighboring bulk material at significantly large deformations. In the context of contact mechanics, engineering the macroscopic interface response requires an ability to tune the friction signal. In particular, bilaterally textured contact interfaces can display strong microscopic interlocking during sliding which manifests itself through oscillations in the macroscopic friction signal about a mean value which can be considerably higher than the microscopic friction coefficient. Hence, it is desirable to be able to control both the mean value and the oscillation amplitude of the friction signal. Although mechanisms for controlling the mean value exist and are readily applicable, tuning the oscillation magnitude while preserving the mean is challenging. In this work, based on a computational contact homogenization framework for soft interfaces, such a mechanism has been proposed which relies on the interaction of 


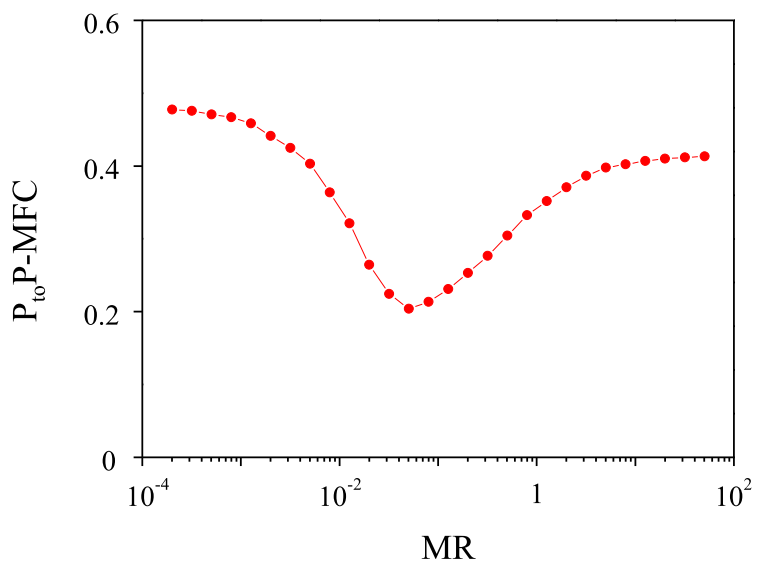

(a) $\mathrm{P}_{\text {to }} \mathrm{P}-\mathrm{MFC}$ for varying $\mathrm{MR}$

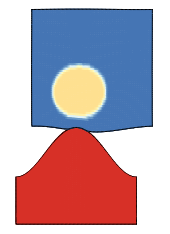

$\mathrm{MR}=8$

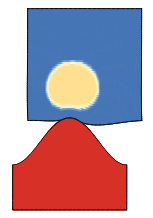

$\mathrm{MR}=0.8$

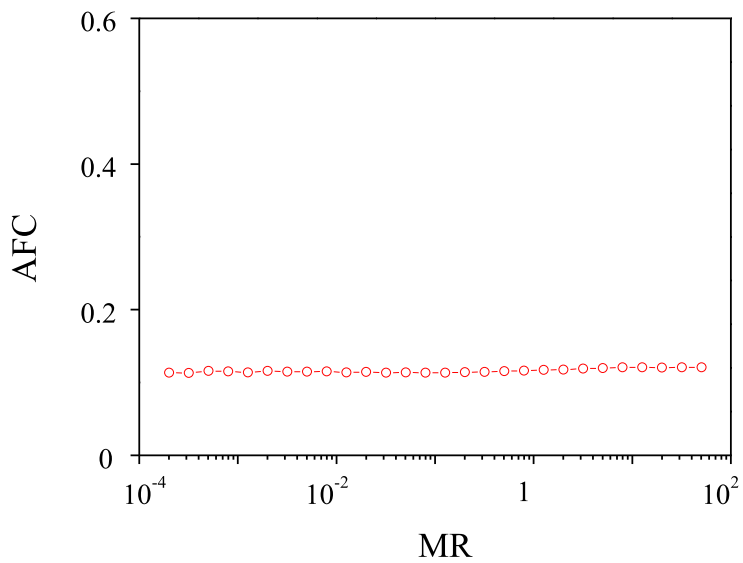

(b) AFC for varying MR

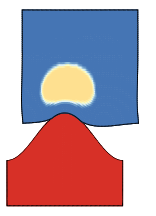

$\mathrm{MR}=0.08 \quad \mathrm{MR}=0.008$

(c) Computation setups at the same simulation instances and with different MR values

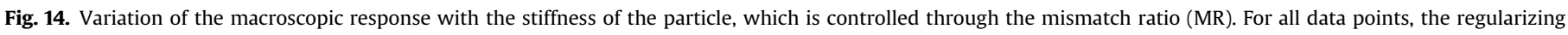
effect of inertia was employed with a scaled density.

surface heterogeneities, which are represented by texture features, with bulk heterogeneities, which are represented by particles embedded beneath the micro-textured surface. It has been shown that this mechanism may be explained on the basis of a superposition approach which can predict the coupled response of combined surface and bulk heterogeneities via an analysis of individual heterogeneity influences in decoupled settings. This tuning approach that is unique to soft interfaces which are easily and highly deformable has been investigated extensively by probing the influence of major micromechanical parameters on the macroscopic frictional response. Specifically, it has been demonstrated that optimal subsurface particle positioning can deliver oscillation magnitude minima such that the manifestations of microscopic interlocking which are macroscopically reminiscent of stick-slip type behavior are hindered to a large extent. Although these investigations have been limited to specific texture and particle geometries in a two-dimensional setting with purely hyperelastic material models, the presented results are indicative of the potential of employing combined bulk and surface heterogeneities towards the tuning of the macroscopic friction signal in a broader range of scenarios. The computational and experimental investigation of more advanced settings will contribute to successful engineering of the macroscopic frictional response in the context of soft interface tribology.

\section{Acknowledgment}

Support for this work was provided by the Scientific and Technological Research Council of Turkey (TÜBITAK) under the 1001 Programme (Grant No. 114M406).

\section{References}

[1] Le Gal A, Klüppel A. Investigation and modelling of rubber stationary friction on rough surfaces. J Phys Condens Matter 2008;20:015007.

[2] Maegawa S, Itoigawa F, Nakamura T. Effect of normal load on friction coefficient for sliding contact between rough rubber surface and rigid smooth surface. Tribol Int 2015;92:335-43.

[3] Momozono S, Takeuchi H, Iguchi Y, Nakamura K, Kyogoku K. Dissipation characteristics of adhesive kinetic friction on amorphous polymer surfaces. Tribol Int 2012;48:122-7.

[4] Fagiani R, Massi F, Chatelet E, Costes JP, Berthier Y. Contact of a finger on rigid surfaces and textiles: friction coefficient and induced vibrations. Tribol Lett 2012;48:145-58.

[5] Adams MJ, Johson SA, Lefevre P, Levesque V, Hayward V, Andre T, et al. Finger pad friction and its role in grip and touch. J R Soc Interface 2013;10:20120467.

[6] Koç İM, Aksu C. Tactile sensing of constructional differences in fabrics with a polymeric finger tip. Tribol Int 2013;59:339-49.

[7] Ella S, Formagne P-Y, Koutsos V, Blackford JR. Investigation of rubber friction on snow for tyres. Tribol Int 2013;59:292-301.

[8] Lahayne O, Pichler B, Reihsner R, Eberhardsteiner J, Suh J, Kim D, et al. Rubber friction on ice: Experiments and modeling. Tribol Lett 2016;62:17.

[9] Maegawa S, Itoigawa F, Nakamura T. Dynamics in sliding friction of soft adhesive elastomer: Schallamach waves as a stress-relaxation mechanism. Tribol Int 2016;96:23-30.

[10] Bai Y, Jagota A, Hui C-Y. Frictional auto-roughening of a surface with spatially varying stiffness. Soft Matter 2014;10:2169-77.

[11] Murarash B, Itovich Y, Varenberg M. Tuning elastomer friction by hexagonal surface patterning. Soft Matter 2011:7:5553-7.

[12] Han L, Yin J, Wang L, Chia K-K, Cohen RE, Rubner MF, et al. Tunable stimulusresponsive friction mechanisms of polyelectrolyte films and tube forests. Soft Matter 2012;8:8642-50.

[13] Cho Y, Kim G, Cho Y, Lee SY, Minsky H, Turner KT, et al. Orthogonal control of stability and tunable dry adhesion by tailoring the shape of tapered nanopillar arrays. Adv Mater 2015;27:7788-93.

[14] Zhou M, Pesika N, Zeng H, Wan J, Zhang X, Meng Y, et al. Design of geckoinspired fibrillar surfaces with strong attachment and easy-removal properties: a numerical analysis of peel-zone. J R Soc Interface 2012;9:2424-36.

[15] Xia SM, Ponson L, Ravichandran G, Bhattacharya K. Adhesion of heterogeneous thin films-II: adhesive heterogeneity. J Mech Phys Solids 2015;83:88-103.

[16] Piccardo M, Chateauminois A, Fretigny C, Pugno NM, Sitti M. Contact compliance effects in the frictional response of bioinspired fibrillar adhesives. J R Soc Interface 2013;10:20130182.

[17] Shen L, Glassmaker NJ, Jagota A, Hui C-Y. Strongly enhanced static friction 
using a film-terminated fibrillar interface. Soft Matter 2008;4:618-25.

[18] Carbone G, Lorenz B, Persson BNJ, Wohlers A. Contact mechanics and rubber friction for randomly rough surfaces with anisotropic statistical properties. Eur Phys J E 2009;29:275-84.

[19] Le Gal A, Guy L, Orange G, Bomal Y, Klüppel M. Modelling of sliding friction for carbon black and silica filled elastomers on road tracks. Wear 2008;2008:60615.

[20] Pan X-D. Contribution of fine filler particles to energy dissipation during wet sliding of elastomer compounds on a rough surface. J Phys D Appl Phys 2007;40:4657-67.

[21] Uchiyama Y, Wada N, Iwai T, Ueda S, Sado S. Friction of short-fiber-reinforced rubber on wet surfaces. J Appl Polym Sci 2005;95:82-9.

[22] Delhaye B, Philippe L, Thonnard J-L. Dynamics of fingertip contact during the onset of tangential slip. J R Soc Interface 2014;11:20140698.

[23] Loskill P, Puthoff J, Wilkinson M, Mecke K, Jacobs K, Autumn K. Macroscale adhesion of gecko setae reflects nanoscale differences in subsurface composition. J R Soc Interface 2012:10:20120587.

[24] Bode K, Ostermeyer G-P. A comprehensive approach for the simulation of hea and heat-induced phenomena in friction materials. Wear 2014;311:47-56.

[25] Peillex G, Baillet L, Berthier Y. Homogenization in non-linear dynamics due to frictional contact. Int J Solids Struct 2008;45:2451-69.

[26] Xia SM, Ponson L, Ravichandran G, Bhattacharya K. Adhesion of heterogeneous thin films-I: elastic heterogeneity. J Mech Phys Solids 2013;61:838-51.

[27] Maegawa S, Itoigawa F, Nakamura T. Effects of stress distribution at the contact interface on static friction force: numerical simulation and model experiment. Tribol Lett 2016;62:15.

[28] Kligerman Y, Varenberg M. Elimination of stick-slip motion in sliding of split or rough surface. Tribol Lett 2014;53:395-9.

[29] Klüppel M, Heinrich G. Rubber friction on self-affine road tracks. Rubber Chem. Technol, 2000:73:578-606.

[30] Persson BNJ. Theory of rubber friction and contact mechanics. J Chem Phys
$2001 ; 115: 3840-61$

[31] De Lorenzis L, Wriggers P. Computational homogenization of rubber friction on rough rigid surfaces. Comput Mater Sci 2013;77:264-80.

[32] Stupkiewicz S, Lewandowski MJ, Lengiewicz J. Micromechanical analysis of friction anisotropy in rough elastic contacts. Int J Solids Struct 2014;51:393143.

[33] Temizer İ. Computational homogenization of soft matter friction: isogeometric framework and elastic boundary layers. Int J Numer Methods Eng 2014;100:953-81.

[34] Temizer İ. Sliding friction across the scales: thermomechanical interactions and dissipation partitioning. J Mech Phys Solids 2016;89:126-48.

[35] Temizer I, Wriggers P. A multiscale contact homogenization technique for the modeling of third bodies in the context interface. Comput Methods Appl Mech Eng 2008;198:377-96.

[36] Temizer I, Wriggers P. Inelastic analysis of granular interfaces via computational contact homogenization. Int J Numer Methods Eng 2010;84:883-915.

[37] Wagner P, Wriggers P, Klapproth C, Prange C, Wies B. Multiscale FEM approach for hysteresis friction of rubber on rough surfaces. Comput Methods Appl Mech Eng 2015;296:150-68.

[38] Wriggers P, Reinelt J. Multi-scale approach for frictional contact of elastomers on rough rigid surfaces. Comput Methods Appl Mech Eng 2009;198:19962009.

[39] Scaraggi M, Comingio D, Al-Qudsi A, Lorenzis L De. The influence of geometrical and rheological non-linearity on the calculation of rubber friction. Tribol Int 2016;101:402-13.

[40] Scaraggi M, Carbone G. A two-scale approach for lubricated soft-contact modeling: an application to lip-seal geometry. Adv Tribol 2012;2012:412190.

[41] Maegawa S, Itoigawa F, Nakamura T. Effect of surface grooves on kinetic friction of a rubber slider. Tribol Int 2016;102:326-32.

[42] Maegawa S, Itoigawa F, Nakamura T. A role of friction-induced torque on sliding friction of rubber materials. Tribol Int 2016:93:182-9. 